\title{
Energies and damping rates of elementary excitations in spin-1 Bose-Einstein condensed gases
}

\author{
Gergely Szirmai \\ Department of Physics of Complex Systems, Roland Eötvös University, \\ Pázmány Péter sétány 1/A, Budapest, H-1117 and \\ Research Group for Statistical Physics of the Hungarian Academy of Sciences, Pázmány Péter Sétány 1/A, Budapest, H-1117 \\ Péter Szépfalusy \\ Department of Physics of Complex Systems, Roland Eötvös University, \\ Pázmány Péter sétány 1/A, Budapest, H-1117 and \\ Research Institute for Solid State Physics and Optics of the Hungarian Academy of Sciences, Budapest, P.O.Box 49, H-1525 \\ Krisztián Kis-Szabó \\ Department of Physics of Complex Systems, Roland Eötvös University, Pázmány Péter sétány 1/A, Budapest, H-1117
}

(Dated: May 9, 2019)

\begin{abstract}
Finite temperature Green's function technique is used to calculate the energies and damping rates of elementary excitations of the homogeneous, dilute, spin-1 Bose gases below the Bose-Einstein condensation temperature both in the density and spin channels. For this purpose the self-consistent dynamical Hartree-Fock model is formulated, which takes into account the direct and exchange processes on equal footing by summing up certain classes of Feynman diagrams. The model is shown to fulfil the Goldstone theorem and to exhibit the hybridization of one-particle and collective excitations correctly. The results are applied to the gases of ${ }^{23} \mathrm{Na}$ and ${ }^{87} \mathrm{Rb}$ atoms.
\end{abstract}

PACS numbers: 03.75.Mn, 03.75.Kk, 03.75Hh

\section{INTRODUCTION}

The experimental realization of Bose-Einstein condensation with ultracold, dilute, alkali gases in magnetic traps $[1,2]$ made it possible, among other things, to meaningfully compare the results of experiment and theory. The reason lies in the diluteness of the gas sample, so the interaction between the atoms of the gas can be modeled by a week pseudopotential, in most of the cases, which can be considered as perturbation in calculations. With the development of optical traps it became possible to confine not just one or two of the magnetic states $|f, m\rangle$ of the atom (the weak-field seeking states), but all of them for a given hyperfine spin $f$ giving rise to a so called spinor condensate [3-7]. Because of these internal degrees of freedom such systems can have much wider dynamical properties than those confined in magnetic traps. The theoretical study of spinor Bose condensed systems was initiated by $[8,9]$.

There are two different phases of spin-1 systems in the presence of a Bose condensate distinguished according to the total magnetic moment of the system $[8,9]$. Namely, the ferromagnetic phase, which is characterized by the existence of a macroscopic magnetic moment and the polar phase where the system has no macroscopic magnetic moment at all. In the absence of an external magnetic field the type of the phase of the spin-1 Bose system depends only on the interaction potential of the atoms, which can be accurately characterized by s-wave scattering lengths $a_{0}$ and $a_{2}$ responsible for scatterings in the total hyperfine spin channel zero and two, respectively [8]. If $a_{2}>a_{0}$ the system prefers the polar phase, while in the case of $a_{2}<a_{0}$ the system is ferromagnetic [8]. For both cases there exist species of alkali Bose atoms. For example the $f=1$ hyperfine spin state of ${ }^{23} \mathrm{Na}$ realizes the polar phase, while that of ${ }^{87} \mathrm{Rb}$ the ferromagnetic phase. Since in the absence of a magnetic field the phase of the system does not depend on experimentally tunable parameters, one can use the word 'case' instead of 'phase'.

The elementary excitations of dilute Bose gases have been studied for a long time [10,11], since these gases represent the simplest prototypes of symmetry breaking quantum systems. The zero temperature elementary excitations of these systems are described reasonably well by the Bogoliubov Hamiltonian [10]. It is not straightforward to generalize Bogoliubov's theory to finite temperatures in a consistent way [12-19]. The simplest finite temperature approach is the self-consistent Hartree model, also known as the random phase approximation $[13,16,19]$.

The inclusion of exchange processes is not trivial in a consistent way using the finite temperature Green's function technique as shown in [17] for a scalar Bose-gas. (See for a formulation of the Hartree-Fock theory for such systems giving the density response functions [20]) For instance self-consistency has proven to be essential for developing a theory satisfying general requirements. As a matter of fact applying unperturbed (i.e. free particle) propagators as internal line contributions leads to an instability for long wavelength fluctuations in case of repulsive interactions. (Note, however, that for attractive interactions such model has been used to describe the collapse of the gas phase [21].) 
In the present paper we generalize the model presented in [17] with systems with scalar condensate to the spin-1 Bose gas. Throughout the paper we will work in the orderd phase. We start from the self-consistent HartreeFock approximation for the equation of state, whose iteration we reveal which contributions are included in the self-consistent procedure. On this basis the self-energies of various one-particle Green's functions can be given in harmony with the equation of state. Furthermore the obtained self-energies fulfill the Goldstone theorem, which is the consequence of the breaking of the gauge and spin rotation symmetries. This ensures gapless excitation spectra. Having determined the one-particle propagators, the expressions of various correlation funcions will be given. The requirement of the coincidence of the one-particle and certain collective excitations is satisfied and the model resulted fits into the dielectric formalism. (For a detailed discussion of the dielectric formalism for spin-1 systems see [19] and for a general review [13].) One has to point out that the model developed can be regarded as an improved mean-field one and as such looses its validity near the phase transition point.

Since the present work deals with homogeneous systems, the results are applicable to trapped gases only when a local density approximation is relevant or when the quantities in question are not sensitive to the finiteness of the system. In analogy with what proved to be the situation in the case of gases with a scalar condensate (see [17, 22] and references therein) one can hope that the damping of the elementary excitations belongs to the latter category. Concerning the local density approach it remains to be seen whether such experiment which provided the frequency of local excitations [23] can be carried out for spin-1 gases. Anyhow, we evaluate the characteristics of the excitations for parameter values which might be relevant to applications. Finally we note that a generalization of the model to finite systems is straightforward like for systems with a scalar condensate [22, 24, 25].

We hope to carry out the calculation directly to trapped systems in the future. At this point one has to emphasize again the self-consistent nature of the model which is very important for applications to confined gases.

The outline of the paper is as follows. In Sec. II. the formulation of the Hartree-Fock approximation is given for the spin-1 Bose gas. It includes the specification of the Hamiltonian, the dealing with the symmetry breaking by a canonical transformation, the equation of state, the definitions and properties the Green's functions and of various correlation functions. In this section we specify what building blocks are to be used for the determination of the various quantities such that

- being consistent with the implicit summation occurring in the Hartree-Fock propagator due to the selfconsistency condition,

- fullfilling the hybridization property.

In Sec. III. the Hartree-Fock model is applied to a ferromagnetic system, the gas of ${ }^{87} \mathrm{Rb}$ atoms [Sec. III A], and to the ${ }^{23} \mathrm{Na}$ gas, which realizes the polar phase [Sec. III B]. Sec. IV. is devoted for further discussion also focusing on an overview of the physical properties of the various collective modes considered in Sec. III. Some details are relegated to the Appendix.

\section{FORMULATION}

We consider a system of spin- 1 bosons in a unit volume box with periodic boundary conditions. For such a system the plausible basis set of one particle states to build the Fock-space from are plane wave states. The creation and destruction operators are $a_{r}^{\dagger}(\mathbf{k})$ and $a_{r}(\mathbf{k})$, which create and annihilate a one-particle state with momentum $\mathbf{k}$ and spin projection $r$, respectively. Throughout the paper Roman indices are used for distinguishing between spin components of a quantity. These indices will always take values from the set $\{+, 0,-\}$ referring to the eigenvalue of $F_{z}$. Unless otherwise stated we understand automatic summation over repeated Roman indices. For our purposes, while the temperature of the system is low, and the gas is very dilute, the system can be well described by the following low energy, effective, grand-canonical Hamiltonian [8]:

$$
\mathcal{H}=\sum_{\mathbf{k}}\left(e_{\mathbf{k}}-\mu\right) a_{r}^{\dagger}(\mathbf{k}) a_{r}(\mathbf{k})+\frac{1}{2} \sum_{\mathbf{k}_{1}+\mathbf{k}_{2}=\mathbf{k}_{3}+\mathbf{k}_{4}} a_{r^{\prime}}^{\dagger}\left(\mathbf{k}_{1}\right) a_{r}^{\dagger}\left(\mathbf{k}_{2}\right) V_{r s}^{r^{\prime} s^{\prime}} a_{s}\left(\mathbf{k}_{3}\right) a_{s^{\prime}}\left(\mathbf{k}_{4}\right),
$$

where $e_{\mathbf{k}}=\hbar^{2} k^{2} / 2 M$ is the kinetic energy of a particle with mass $M$, and $\mu$ is the chemical potential. We restrict ourselves to the Hartree-Fock approximation, and for such purposes the interatomic potential in Eq. (1) can be modeled by the momentum independent pseudopotential $[12,26]$ :

$$
V_{r s}^{r^{\prime} s^{\prime}}=c_{n} \delta_{r s} \delta_{r^{\prime} s^{\prime}}+c_{s}(\mathbf{F})_{r s}(\mathbf{F})_{r^{\prime} s^{\prime}}
$$


with parameters:

$$
\begin{aligned}
& c_{n}=\frac{4 \pi \hbar^{2}}{M} \frac{a_{0}+2 a_{2}}{3}, \\
& c_{s}=\frac{4 \pi \hbar^{2}}{M} \frac{a_{2}-a_{0}}{3} .
\end{aligned}
$$

The parameters $a_{0}$ and $a_{2}$ are the scattering length in the total hyperfine spin channel zero and two, respectively. Note, that $c_{s}>0$ for the polar case and $c_{s}<0$ for the ferromagnetic one. In our representation the one-particle spin operators are

$$
F_{x}=\frac{1}{\sqrt{2}}\left[\begin{array}{ccc}
0 & 1 & 0 \\
1 & 0 & 1 \\
0 & 1 & 0
\end{array}\right], \quad F_{y}=\frac{1}{\sqrt{2}}\left[\begin{array}{ccc}
0 & -i & 0 \\
i & 0 & -i \\
0 & i & 0
\end{array}\right], \quad F_{z}=\left[\begin{array}{ccc}
1 & 0 & 0 \\
0 & 0 & 0 \\
0 & 0 & -1
\end{array}\right] .
$$

Let us denote the temperature with $T_{0}$, where the symmetric (uncondensed or high temperature) phase becomes unstable. To treat the system in the Bose condensed phase one can introduce the following new set of operators with a canonical transformation:

$$
\begin{aligned}
& b_{r}(\mathbf{k})=a_{r}(\mathbf{k})-\delta_{\mathbf{k}, 0} \sqrt{N_{0}} \zeta_{r} \\
& b_{r}^{\dagger}(\mathbf{k})=a_{r}^{\dagger}(\mathbf{k})-\delta_{\mathbf{k}, 0} \sqrt{N_{0}} \zeta_{r}^{\dagger} .
\end{aligned}
$$

The $N_{0}$ and $\zeta_{r}$ parameters appearing in the transformation (5) describe the number and spinor of particles in the condensate, respectively. The spinor is normalized to unity. The spinor describing the polar state can be taken as $\zeta=(0,1,0)^{T}$, while the ferromagnetic spinor as $\zeta=(1,0,0)^{T}$. Here and in the following, the ' $T$ ' in the superscript means transposition. The equation of state consists of two parts. The first one is the condition

$$
\left\langle b_{r}(\mathbf{k})\right\rangle=\left\langle b_{r}^{\dagger}(\mathbf{k})\right\rangle=0
$$

which provides an equation among $N_{0}$ and the chemical potential (see e.g. Ref. [19]). The other part is the expression of the particle number. In our case

$$
N=\sum_{\mathbf{k}}\left\langle a_{r}^{\dagger}(\mathbf{k}) a_{r}(\mathbf{k})\right\rangle=N_{0}(T, \mu)+\sum_{\mathbf{k}}\left\langle b_{r}^{\dagger}(\mathbf{k}) b_{r}(\mathbf{k})\right\rangle
$$

where we have used Eqs. (5) to arrive to the right hand side of the above equation.

\section{A. Green's functions}

To calculate the grand-canonical averages in the equation of state (6) we use the method of finite temperature Green's function theory. The Green's functions of the system are defined by:

$$
\mathcal{G}_{\gamma \delta}^{r s}(\mathbf{k}, \tau):=-\left\langle T_{\tau}\left[b_{r}^{\gamma}(\mathbf{k}, \tau) b_{s}^{\delta^{\dagger}}(\mathbf{k}, 0)\right]\right\rangle
$$

where $\tau$ stands for the imaginary time and $T_{\tau}$ is the $\tau$ ordering operator. The Greek indices are introduced for abbreviation, with $b_{r}^{1}(\mathbf{k})=b_{r}(\mathbf{k})$ and $b_{r}^{-1}(\mathbf{k})=b_{r}^{\dagger}(-\mathbf{k})$. Since they show the direction of propagation of the Green's function, we also will refer to them as direction indices. The convention of automatic summation is also understood for the Greek indices unless stated otherwise.

These functions are periodic in $\tau$ with period $\beta \hbar$. It is more convenient to use the Fourier series instead of the imaginary time dependent functions:

$$
\mathcal{G}_{\gamma \delta}^{r s}\left(\mathbf{k}, i \omega_{n}\right)=\frac{1}{\beta \hbar} \int_{0}^{\beta \hbar} d \tau e^{i \omega_{n} \tau} \mathcal{G}_{\gamma \delta}^{r s}(\mathbf{k}, \tau),
$$

with $\omega_{n}=2 n \pi / \beta \hbar$ the Bose discrete Matsubara frequency [27].

The perturbation series of the Green's functions permit a simple partial summation. The Green's functions satisfy the generalized Dyson-Beliaev equations [13, 19, 27]:

$$
\mathcal{G}_{\gamma \delta}^{r s}\left(\mathbf{k}, i \omega_{n}\right)=\mathcal{G}_{(0) \gamma \delta}^{r s}\left(\mathbf{k}, i \omega_{n}\right)+\mathcal{G}_{(0) \gamma \rho}^{r r^{\prime}}\left(\mathbf{k}, i \omega_{n}\right) \Sigma_{\rho \sigma}^{r^{\prime} s^{\prime}}\left(\mathbf{k}, i \omega_{n}\right) \mathcal{G}_{\sigma \delta}^{s^{\prime} s}\left(\mathbf{k}, i \omega_{n}\right),
$$


where

$$
\mathcal{G}_{(0) \gamma \delta}^{r s}\left(\mathbf{k}, i \omega_{n}\right)=\frac{\delta_{r s} \delta_{\gamma \delta}}{i \omega_{n}-\hbar^{-1} e_{\mathbf{k}}}
$$

is the free propagator, and $\Sigma_{\rho \sigma}^{r^{\prime} s^{\prime}}\left(\mathbf{k}, i \omega_{n}\right)$ is the self-energy, i.e. the sum of the contribution of the one particle irreducible Feynman diagrams, which connect to two external lines, one from the left, with indices $r^{\prime}, \rho$, momentum $\mathbf{k}$ and frequency $\omega_{n}$, the other from the right, with indices $s^{\prime}, \sigma$, momentum $k$ and frequency $\omega_{n}$ (we will call the one particle irreducible graphs simply as irreducible graphs). Note, that the chemical potential is included in the self energy instead of the free-proagator (see, e.g., Ref. [19]). Both the Green's functions and self-energies obey the following symmetry properties [19]:

$$
\mathcal{G}_{\gamma \delta}^{r s}\left(\mathbf{k}, i \omega_{n}\right)=\mathcal{G}_{\delta \gamma}^{s r}\left(\mathbf{k}, i \omega_{n}\right)=\mathcal{G}_{-\delta,-\gamma}^{s r}\left(\mathbf{k},-i \omega_{n}\right),
$$

where $\Sigma$ can also stand instead of $\mathcal{G}$ in Eq. (11). Since spin and space variables are not coupled, the Green's functions and self-energies depend only on the modulus of the momentum.

According to the usual procedure elementary excitations can be obtained. Continuing the Green's functions in their frequency variable from the imaginary Matsubara frequencies to the complex plane, requiring to be analytical on the upper-half plane, one arrives at the retarded Green's functions, the poles of which (in the lower half-plane) give the various one-particle elementary excitations of the system.

\section{B. The equation of state}

Now let us return to the problem of determining the equation of state (6) of the symmetry breaking dilute spin-1 Bose gas in the Hartree-Fock approximation. Indeed, the equation of state in the Hartree-Fock approximation is the system of nonlinear equations resulting from the self-consistency condition of the Hartree-Fock propagators along with Eqs. (6).

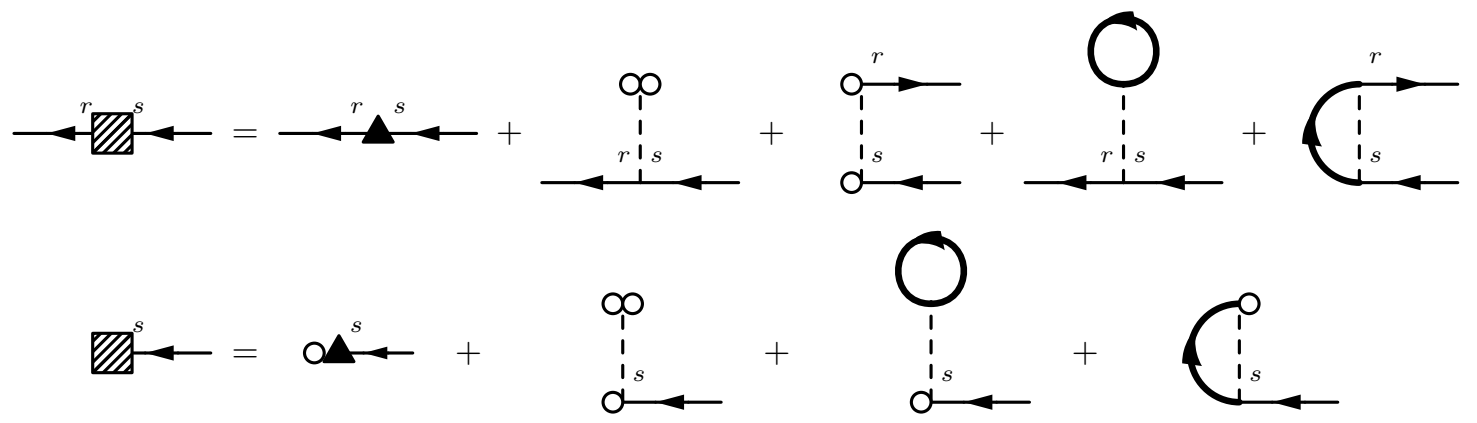

FIG. 1: The Feynman diagrams contributing to the self-energy $\widehat{\Sigma}_{11}^{r s}$ of the internal line (a) and the contribution of the tadpole diagrams (b). The heavy line depicts the internal line, while the thin line corresponds to the free propagator. The circle denotes a condensate operator, the dashed line is for the interaction and the triangle corresponds to $-\mu$.

We denote the Hartree-Fock propagators (which will play the role of internal lines) by $\widehat{\mathcal{G}}_{11}^{r s}\left(\mathbf{k}, i \omega_{n}\right)$ and define it with the following equation:

$$
\widehat{\mathcal{G}}_{11}^{r s}\left(\mathbf{k}, i \omega_{n}\right)=\frac{\delta_{r s}}{i \omega_{n}-\hbar^{-1} e_{\mathbf{k}}-\widehat{\Sigma}_{11}^{r s}}
$$

where $\widehat{\Sigma}_{11}^{r s}$ is the momentum and frequency independent self-energy of the internal line. The Feynman diagrams contributing to the self-energy of the internal line can be seen in Fig. 1. The corresponding equation reads as:

$$
\widehat{\Sigma}_{11}^{r s}=\hbar^{-1}\left[-\mu \delta_{r s}+\left(N_{0} \zeta_{r^{\prime}} \zeta_{s^{\prime}}+H^{r^{\prime} s^{\prime}}\right)\left(V_{r s}^{r^{\prime} s^{\prime}}+V_{s^{\prime} s}^{r r^{\prime}}\right)\right],
$$

where $H^{r^{\prime} s^{\prime}}$ is the contribution of the loop (appearing in the Hartree and Fock terms):

$$
H^{r s}=\lim _{\eta \rightarrow 0} \int \frac{d^{3} q}{(2 \pi)^{3}} \frac{1}{\beta \hbar} \sum_{i \nu_{n}}\left[-\widehat{\mathcal{G}}_{11}^{r s}\left(\mathbf{q}, i \nu_{n}\right)\right] e^{i \nu_{n} \eta}=\frac{\delta_{r s}}{(2 \pi)^{2} \lambda^{3}} \Gamma(3 / 2) F\left(3 / 2, \beta \hbar \widehat{\Sigma}_{11}^{r r}\right),
$$


with

$$
\lambda=\frac{\hbar}{\sqrt{2 M k_{B} T}}
$$

the thermal wavelength, $\Gamma(s)$ the gamma function and $F(s, \gamma)$ the Bose-Eintein integral with parameter $s$ and argument $\gamma[28]$.

The value of the chemical potential is determined by the requirement Eq. (6a), or equivalently by the vanishing of the contribution of tadpole diagrams [19]:

$$
0=\Sigma_{01}^{s}=\hbar^{-1} \sqrt{N_{0}} \zeta_{r}\left[-\mu \delta_{r s}+N_{0} \zeta_{r^{\prime}} \zeta_{s^{\prime}} V_{r s}^{r^{\prime} s^{\prime}}+H^{r^{\prime} s^{\prime}}\left(V_{r s}^{r^{\prime} s^{\prime}}+V_{s^{\prime} s}^{r^{\prime} r}\right)\right] .
$$

Since we work in the ordered phase, $N_{0} \neq 0$ everywhere assumed. The simultaneous solution of equations (12), (13), (14), (16) (which is the consequence of Eq. (6a)), and Eq. (6b) provides the self-energy and propagator of the internal lines.

For a ferromagnetic system the condensate spinor can be chosen to be $\zeta_{r}=(1,0,0)^{T}$. According to Eq. (16) the chemical potential for this case reads as:

$$
\mu=c_{n}\left(N_{0}+2 H^{++}+H^{00}+H^{--}\right)+c_{s}\left(N_{0}+2 H^{++}+H^{00}-H^{--}\right)
$$

while from Eqs. (13) and (17) the self-energies take the following form:

$$
\begin{aligned}
\hbar \widehat{\Sigma}_{11}^{++} & =\left(c_{n}+c_{s}\right) N_{0}, \\
\hbar \widehat{\Sigma}_{11}^{00} & =-\left(c_{n}+c_{s}\right) H^{++}+\left(c_{n}-c_{s}\right) H^{00}+2 c_{s} H^{--}, \\
\hbar \widehat{\Sigma}_{11}^{--} & =-\left(c_{n}+3 c_{s}\right) H^{++}+\left(c_{n}+3 c_{s}\right) H^{--}-2 c_{s} N_{0} .
\end{aligned}
$$

The contributions of the single loops are also related to the self-energies through Eq. (14). There is a final equation from Eq. (6b) reading as:

$$
N=N_{0}+H^{++}+H^{00}+H^{--} .
$$

The system of equations (17), (18), (14) and (19) form the equation of state in the following way. One can express the condensate density with the help of $N, H^{++}, H^{00}, H^{--}$from Eq. (19) and substitute it to Eqs. (18). These equations now form a closed set of equations toether with Eq. (14). One can solve them for $H^{++}, H^{00}, H^{--}$as functions of $T, N$, and substitute them to Eq. (17) to arrive at the equation of state $\mu=\mu(T, N)$. An alternate way is to express $N_{0}$ from Eq. (17) and solve the equations for the loop terms with $T, \mu$ fixed, and then express $N=N(T, \mu)$. The two equations of state are necessarily equivalent.

For a polar system the condensate spinor can be taken as $\zeta_{r}=(0,1,0)^{T}$. The chemical potential for this phase, from Eq. (16), reads as:

$$
\mu=c_{n}\left(N_{0}+H^{++}+2 H^{00}+H^{--}\right)+c_{s}\left(H^{++}+H^{--}\right) .
$$

The self-energies (13) in this case take the following form:

$$
\begin{aligned}
\hbar \widehat{\Sigma}_{11}^{00} & =c_{n} N_{0}, \\
\hbar \widehat{\Sigma}_{11}^{++} & =\left(c_{n}-c_{s}\right)\left(H^{++}-H^{00}\right)+c_{s} N_{0},
\end{aligned}
$$

where we have used the specific form of the chemical potential (20) and that $\widehat{\Sigma}_{11}^{++}=\widehat{\Sigma}_{11}^{--}$and $H^{++}=H^{--}$. Equation (6b) can be expressed as:

$$
N=N_{0}+2 H^{++}+H^{00}
$$

Expressing $N_{0}$ from Eq. (22) and substituting it to Eqs. (21) the latter ones can be solved together with Eq. (14) for $H^{00}$ and $H^{++}$. They can be used in Eq. (20) to express the equation of state as $\mu=\mu(T, N)$. The alternate way is also possible here, i.e. to express the total number of particles as $N=N(T, \mu)$.

\section{Effective interactions}

In this subsection we discuss the effective interactions which will play an important role in the following. The self consistent solution of the Hartree-Fock propagators contains a partial summation of infinite number of Feynman graphs. This partial summation is visible from the iterative solution of the Hartree-Fock propagators. With iteration one arrives to effective interactions which are the contributions of repeated exchange and direct terms. 


\section{Repeated exchange interactions}

Denote the contribution of multiple exchange interactions with the quantity $\Theta_{r^{\prime} s^{\prime}}^{r s}\left(k_{1}, k_{2}, q\right)$, defined by the Feynmangraphs in Fig. 2. For the sake of brevity one can introduce the four-vector notation: $q=\left(\mathbf{q}, i \nu_{n}\right)$ and $k=\left(\mathbf{k}, i \omega_{n}\right)$.

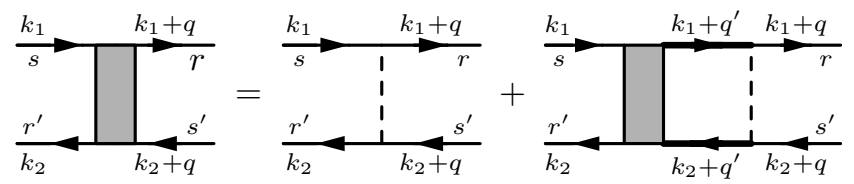

FIG. 2: The Feynman diagrams defining $\Theta_{r^{\prime} s^{\prime}}^{r s}\left(k_{1}, k_{2}, q\right)$. The shaded square depicts $\Theta$.

The equation for $\Theta$ reads as:

$$
\left.\Theta_{r^{\prime} s^{\prime}}^{r s}\left(k_{1}, k_{2}, q\right)=V_{r^{\prime} s^{\prime}}^{r s}+\int D q^{\prime}\left[\Theta_{r^{\prime} b}^{a s}\left(k_{1}, k_{2}, q^{\prime}\right) \rho_{(0)}^{b a}\right)_{c d}^{b a}\left(k_{1}+q^{\prime}, k_{2}+q^{\prime}\right) V_{d s^{\prime}}^{r c}\right],
$$

where

$$
\begin{aligned}
& \int D k \equiv \frac{1}{\beta \hbar} \sum_{i \omega_{n}} \int \frac{d^{3} k}{(2 \pi)^{3}}, \quad \text { and } \\
& \rho_{(0){ }_{r_{1} r_{2}}^{s_{2} s_{1}}\left(k_{1}, k_{2}\right)}:=-\hbar^{-1} \widehat{\mathcal{G}}_{11}^{s_{1} r_{1}}\left(k_{1}\right) \widehat{\mathcal{G}}_{11}^{s_{2} r_{2}}\left(k_{2}\right)
\end{aligned}
$$

were also introduced. The contribution of the bubble graph can be calculated from $\rho_{(0)}$, which we give here for future reference:

$$
\int D q \rho_{(0)}{ }_{r_{1} r_{2}}^{s_{2} s_{1}}(q, k+q)=\Pi_{(0)}{ }_{r_{1} r_{2}}^{s_{2} s_{1}}(k) .
$$

Due to the conservation of the spin z component Eq. (23) decouples to independent matrix equations. Let us define the following matrices for 0 spin transfer:

$$
\begin{aligned}
{ }^{0} \underline{\underline{\Theta}}= & {\left[\begin{array}{ccc}
\Theta_{++}^{++} & \Theta_{++0}^{0+} & \Theta_{+-}^{-+} \\
\Theta_{0+}^{+0} & \Theta_{00}^{00} & \Theta_{0-}^{-0} \\
\Theta_{-+}^{+-} & \Theta_{-0}^{0-} & \Theta_{--}^{--}
\end{array}\right], } \\
{ }^{0} \underline{\underline{V}}= & {\left[\begin{array}{ccc}
c_{n}+c_{s} & c_{s} & 0 \\
c_{s} & c_{n} & c_{s} \\
0 & c_{s} & c_{n}+c_{s}
\end{array}\right], } \\
{ }^{0} \underline{\rho}_{(0)}= & {\left[\begin{array}{ccc}
\rho_{(0)}^{++} & 0 & 0 \\
0 & \rho_{(0)} 00 & 0 \\
0 & 0 & \rho_{(0)}^{--}
\end{array}\right] . }
\end{aligned}
$$

For +1 spin transfer one defines

$$
\begin{aligned}
{ }^{+} \underline{\underline{\Theta}} & =\left[\begin{array}{cc}
\Theta_{00}^{++} & \Theta_{0-}^{0+} \\
\Theta_{-0}^{+0} & \Theta_{--}^{00}
\end{array}\right], \\
{ }^{+} \underline{\underline{V}} & =\left[\begin{array}{cc}
c_{n} & c_{s} \\
c_{s} & c_{n}
\end{array}\right], \\
{ }^{+} \underline{\rho}_{(0)} & =\left[\begin{array}{cc}
\rho_{(0)}^{0+} & 0 \\
0 & \rho_{(0)_{0-}^{-0}}
\end{array}\right] .
\end{aligned}
$$

And finally, for +2 spin transfer one introduces

$$
\begin{gathered}
{ }^{Q} \underline{\underline{\Theta}}=\Theta_{--}^{++}, \\
{ }^{Q} \underline{\underline{V}}=c_{n}-c_{s}, \\
{ }^{Q} \underline{\rho}_{(0)}=\rho_{(0)_{+-}^{-+} .}
\end{gathered}
$$


With the definitions (26), (27) and (28) one can cast Eq. (23) to the following integral equations for $n \in\{0,+, Q\}$ :

$$
\int D q^{\prime} \stackrel{n}{\underline{\Theta}}\left(k_{1}, k_{2}, q^{\prime}\right)\left[\delta\left(q-q^{\prime}\right) \underline{\underline{1}}-{ }^{n} \underline{\underline{\rho}}_{(0)}\left(k_{1}+q^{\prime}, k_{2}+q^{\prime}\right){ }^{n} \underline{\underline{V}}\right]={ }^{n} \underline{\underline{V}} .
$$

The $\delta$-function is normalized to unity with the definition

$$
\delta(k) \equiv(2 \pi)^{3}(\beta \hbar) \delta^{(3)}(\mathbf{k}) \delta_{\omega_{n}, 0} .
$$

It is straightforward to check that the solution of Eq. (29) reads as:

$$
{ }^{n} \underline{\underline{\Theta}}\left(k_{1}, k_{2}, q\right)={ }^{n} \underline{\underline{V}} \cdot{ }^{n} \underline{\underline{\varepsilon}}^{\star-1}\left(k_{2}-k_{1}\right),
$$

with

$$
\underline{\underline{\varepsilon}}^{\star}(k)=\underline{\underline{1}}-{ }^{n} \underline{\underline{\Pi}}_{(0)}(k)^{n} \underline{\underline{V}}
$$

The matrix ${ }^{n} \underline{\underline{\Pi}}_{(0)}$ is understood as the integral of the matrix ${ }^{n} \underline{\underline{\rho}}_{(0)}$ according to Eq. (25). The matrices ${ }^{n} \underline{\underline{\varepsilon}}^{\star}$ can be interpreted as dielectric functions with respect to repeated exchange interactions. It is important to note that ${ }^{n} \underline{\underline{\Theta}}\left(k_{1}, k_{2}, q\right)$ does not depend on the amount of momentum transferred $(q)$ at all, as is visible from Eq. (31a).

\section{Repeated direct interactions}

For the determination of the effective interaction representing multiple direct interactions, first consider the regular polarization functions, defined by the Feynman diagrams depicted in Fig. 3, or with the following equation (already separated by spin transfer):

$$
{ }^{n} \underline{\underline{\Pi}}^{(r)}(k)={ }^{n} \underline{\underline{\Pi}}_{(0)}(k)+\int D q D q^{\prime} \stackrel{n}{\underline{\rho}}_{(0)}(q, k+q){ }^{n} \underline{\underline{\Theta}}\left(q, k+q, q^{\prime}-q\right){ }^{n} \underline{\underline{\rho}}_{(0)}\left(q^{\prime}, k+q^{\prime}\right),
$$

which leads to

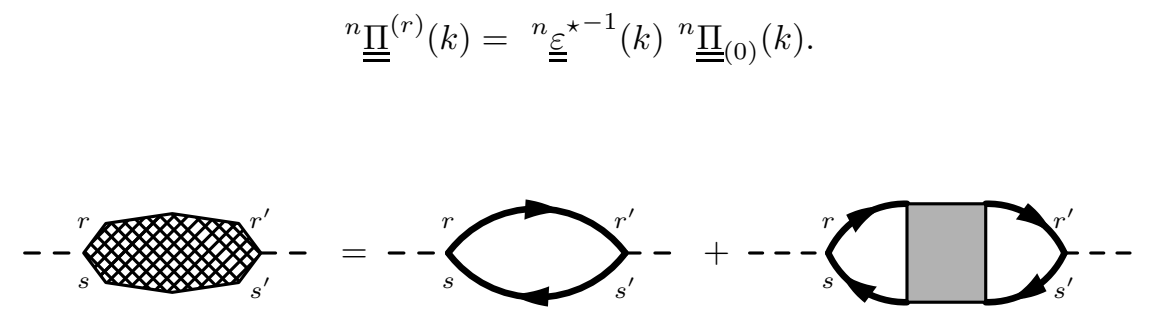

FIG. 3: The Feynman diagrams defining the regular polarization $\Pi_{r^{\prime} s^{\prime}}^{(r) s r}$. The hatched poligon represents the regular polarization function.

The direct effective interaction is then defined by the Feynman diagrams in Fig. 4, or by the equation

$$
W_{r^{\prime} s^{\prime}}^{r s}(k)=V_{r^{\prime} s^{\prime}}^{r s}+W_{a b}^{r s}(k) \Pi_{c d}^{(r) b a}(k) V_{r^{\prime} s^{\prime}}^{d c} .
$$

Equation (34) splits into separate matrix equations according to the amount of spin transferred. Introducing the quantities:

$$
\begin{array}{rlrl}
{ }^{0} \underline{\underline{W}} & =\left[\begin{array}{ccc}
W_{+}^{++} & W_{00}^{++} & W_{--}^{++} \\
W_{++}^{00} & W_{00}^{00} & W_{--}^{00} \\
W_{++}^{-} & W_{00}^{--} & W_{--}^{--}
\end{array}\right], & { }^{0} \underline{\underline{C}} & =\left[\begin{array}{ccc}
c_{n}+c_{s} & c_{n} & c_{n}-c s \\
c_{n} & c_{n} & c_{n} \\
c_{n}-c_{s} & c_{n} & c_{n}+c_{s}
\end{array}\right], \\
{ }^{+} \underline{\underline{W}} & =\left[\begin{array}{ll}
W_{+0}^{0+} & W_{0-}^{0+} \\
W_{+0}^{-0} & W_{0-}^{-0}
\end{array}\right], & { }^{+} \underline{\underline{C}} & =\left[\begin{array}{cc}
c_{s} & c_{s} \\
c_{s} & c_{s}
\end{array}\right], \\
{ }^{Q} \underline{\underline{\underline{W}}} & =W_{+-}^{-+}, & { }^{Q} \underline{\underline{C}}=0,
\end{array}
$$




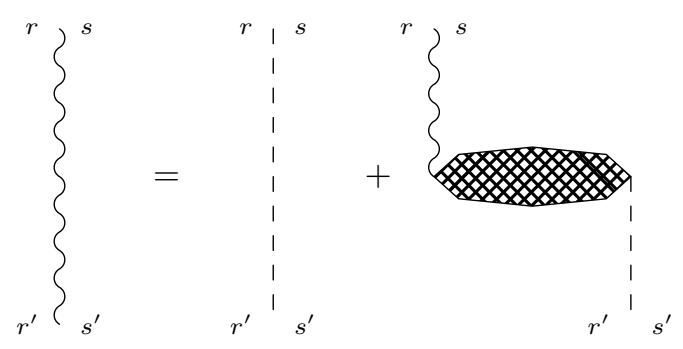

FIG. 4: The Feynman diagrams defining the direct effective interaction. The wiggly line represents the direct effective interaction.

the resulting equations are

$$
{ }^{n} \underline{\underline{W}}(k)={ }^{n} \underline{\underline{C}}+{ }^{n} \underline{\underline{W}}(k) \stackrel{n}{\underline{\Pi}^{(r)}}(k){ }^{n} \underline{\underline{C}}
$$

for $n \in\{0,+, Q\}$. From ${ }^{Q} \underline{\underline{C}}=0$ it immediately follows that ${ }^{Q} \underline{\underline{W}}=0$.

The solutions of Eq. (36) reads as:

$$
{ }^{n} \underline{\underline{W}}(k)={ }^{n} \underline{\underline{C}}{ }^{n} \underline{\underline{\varepsilon}}^{(r)}{ }^{-1}(k),
$$

with

$$
{ }^{n} \underline{\underline{\varepsilon}}(r)={ }^{n} \underline{\underline{1}}-{ }^{n} \underline{\underline{\underline{\Pi}}}{ }^{(r)}{ }^{n} \underline{\underline{C}}
$$

3. The equation of state in terms of the effective interactions

The final structure of the Hartree-Fock self-energies and tadpole diagrams, as a result of the iteration of the HartreeFock propagator, are depicted in Fig. 5. a) and b), respectively, with the help of the effective interactions. For the sake of simplicity we have omitted the spin indices from the figure. Note, that in the final structure of the self-energies in Fig. 5. a) the Hartree-Fock propagators do not join vertices of the same interaction line, such propagators are always unperturbed ones. The iteration, of course, can be continued by substituting the remaining Hartree-Fock propagators with self-energies given in Fig. 1 a) in the self-energies, a procedure without end. These further iterations, however, do not generate new blocks, i.e. the building blocks remain the effective interactions $\Theta$ and $W$.

\section{Anomalous vertex functions}

For the sake of a simplified notation it is convenient to introduce the anomalous vertex functions $\widetilde{\Lambda}_{a \alpha}^{s r}(k)$, and $\widetilde{\Lambda}_{r^{\prime} s^{\prime}}^{a \alpha}(k)$, whose general definitions can be found in [19]. In the Hartree-Fock approximation the Feynman diagrams for $\widetilde{\Lambda}_{a, 1}^{s r}$ are depicted in Fig. 6. The equations for the anomalous vertices in this approximation read as:

$$
\begin{aligned}
& \widetilde{\Lambda}_{a, 1}^{s r}(k)=\Lambda_{(0) a, 1}^{s r}+\int D q \rho_{(0) c d}^{s r}(q, k+q) \Theta_{d a}^{r^{\prime} c}(q, k+q,-q) \Lambda_{(0) a, 1}^{s^{\prime} r^{\prime}}, \quad \text { with } \quad \Lambda_{(0) a, 1}^{s r}=\delta_{s, a} \zeta_{r} \sqrt{N_{0}}, \\
& \widetilde{\Lambda}_{a,-1}^{s r}(k)=\Lambda_{(0) a,-1}^{s r}+\int D q \rho_{(0) c d}^{s r}(q, k+q) \Theta_{d a}^{r^{\prime} c}(q, k+q,-k-q) \Lambda_{(0) a,-1}^{s^{\prime} r^{\prime}}, \quad \text { with } \quad \Lambda_{(0) a,-1}^{s r}=\delta_{r, a} \zeta_{s} \sqrt{N_{0}}, \\
& \widetilde{\Lambda}_{r^{\prime} s^{\prime}}^{a, 1}(k)=\Lambda_{(0) r^{\prime} s^{\prime}}^{a, 1}+\int D q \Lambda_{(0) r s}^{a, 1} \Theta_{s d}^{c r}(0, k, q) \rho_{(0) r^{\prime} s^{\prime}}^{d c}(q, k+q), \quad \text { with } \quad \Lambda_{(0) r^{\prime} s^{\prime}}^{a, 1}=\delta_{a, s^{\prime}} \zeta_{r^{\prime}} \sqrt{N_{0}}, \\
& \widetilde{\Lambda}_{r^{\prime} s^{\prime}}^{a,-1}(k)=\Lambda_{(0) r^{\prime} s^{\prime}}^{a,-1}+\int D q \Lambda_{(0) r s}^{a,-1} \Theta_{s d}^{c r}(-k, 0, k+q) \rho_{(0) r^{\prime} s^{\prime}}^{d c}(q, k+q), \quad \text { with } \quad \Lambda_{(0) r^{\prime} s^{\prime}}^{a,-1}=\delta_{a, r^{\prime}} \zeta_{s^{\prime}} \sqrt{N_{0}} .
\end{aligned}
$$

Note that in the diagrams for $(39 \mathrm{c})$ and $(39 \mathrm{~d})$ the type of left and right connections are interchanged as compare to the diagrams for (39a) and (39b), respectively. 


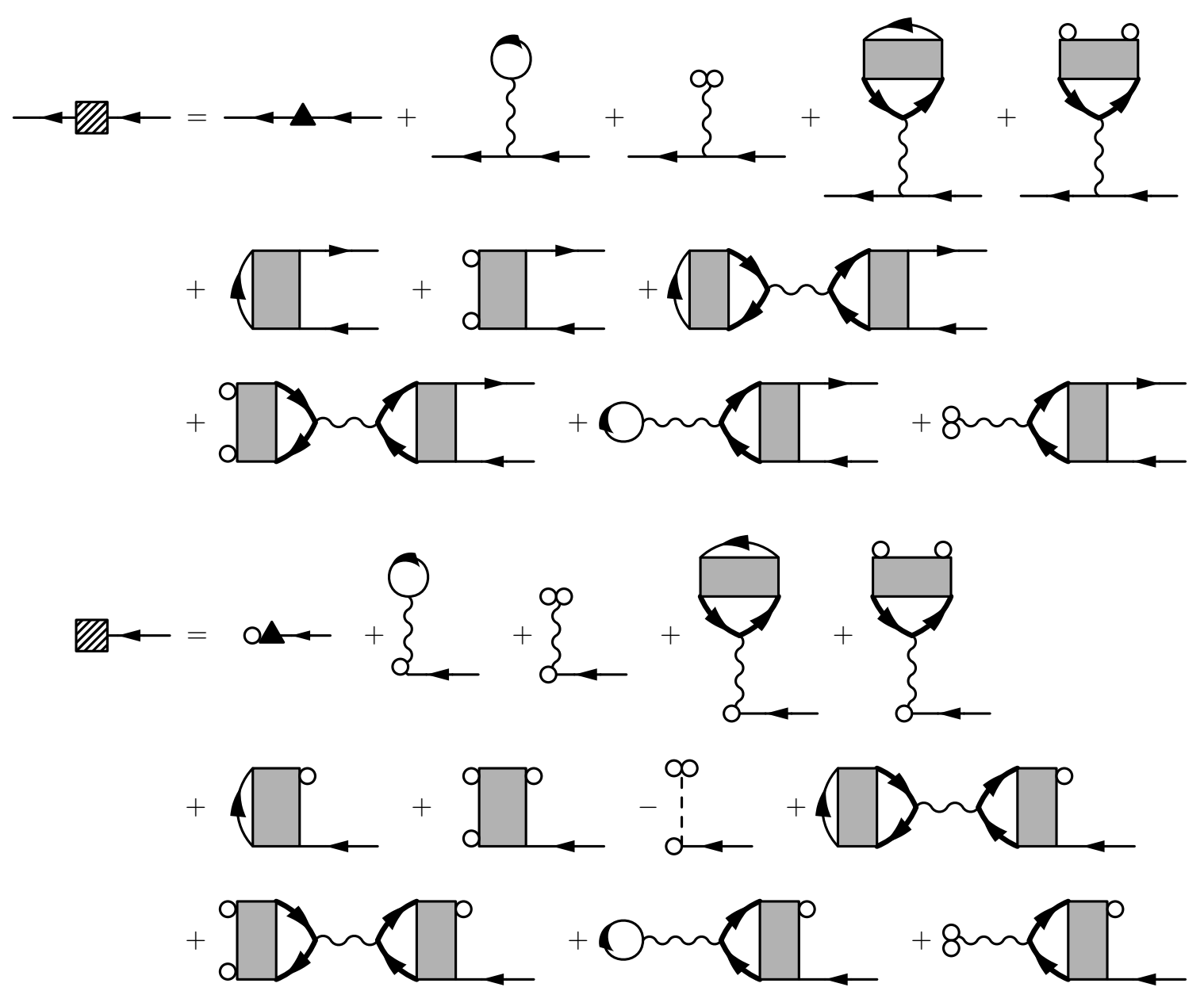

(a)

FIG. 5: The Feynman diagrams contributing to the self-energy $\widehat{\Sigma}_{11}^{r s}$ of the internal line (a), and to the tadpole diagrams (b).

Equations (39) also split to separate equations according to the amount of spin transfered from the right to the left. Let us define

$$
\begin{aligned}
& \underline{\Omega}_{\alpha}^{0}=\left(\begin{array}{c}
\widetilde{\Lambda}_{0 \alpha}^{++} \\
\widetilde{\Lambda}_{0 \alpha}^{00} \\
\widetilde{\Lambda}_{0 \alpha}^{--}
\end{array}\right), \quad \quad \underline{\Lambda}^{0}=\left(\begin{array}{c}
\widetilde{\Lambda}_{\widetilde{\Lambda}^{\alpha+}}^{0 \alpha} \\
\widetilde{\Lambda}_{00}^{0 \alpha} \\
\widetilde{\Lambda}_{---}^{0 \alpha}
\end{array}\right), \\
& +\underline{\tilde{\Lambda}}_{1}=\left(\begin{array}{c}
\widetilde{\Lambda}_{-, 1}^{0+} \\
\widetilde{\Lambda}_{-, 1}^{-0}
\end{array}\right), \quad+\underline{\widetilde{\Lambda}}^{1}=\left(\begin{array}{c}
\widetilde{\Lambda}_{+0}^{-, 1} \\
\widetilde{\Lambda}_{0-1}^{-, 1}
\end{array}\right), \\
& +\underline{\widetilde{\Lambda}}_{-1}=\left(\begin{array}{c}
\widetilde{\Lambda}_{+,-1}^{0+} \\
\widetilde{\Lambda}_{+,-1}^{-0}
\end{array}\right), \quad+\underline{\widetilde{\Lambda}}^{-1}=\left(\begin{array}{c}
\widetilde{\Lambda}_{+0,-1}^{+,-1} \\
\widetilde{\Lambda}_{0-}^{+,-1}
\end{array}\right), \\
& -{ }_{s}^{r} \leftarrow=-{ }_{s}^{r} \mathrm{o}^{a}-+\underbrace{a}_{1}
\end{aligned}
$$

FIG. 6: The Feynman diagrams defining the proper anomalous vertex. The hatched triangle represents the proper anomalous vertex. 
for the polar case and

$$
\begin{array}{rlrl}
{ }^{0} \widetilde{\Lambda}_{\alpha} & =\left(\begin{array}{c}
\widetilde{\Lambda}_{+\alpha}^{++} \\
\widetilde{\Lambda}_{+\alpha}^{00} \\
\widetilde{\Lambda}_{+\alpha}^{+\alpha}
\end{array}\right), & \underline{\Lambda}^{0}=\left(\begin{array}{c}
\widetilde{\Lambda}_{\widetilde{\Lambda}^{++}}^{+\alpha} \\
\widetilde{\Lambda}_{00}^{+\alpha} \\
\tilde{\Lambda}_{--}^{+\alpha}
\end{array}\right), \\
+\underline{\widetilde{\Lambda}}_{1}=\left(\begin{array}{c}
\widetilde{\Lambda}_{0,1}^{0+} \\
\widetilde{\Lambda}_{0,1}^{-0}
\end{array}\right), & +\underline{\widetilde{\Lambda}}^{1}=\left(\begin{array}{c}
\widetilde{\Lambda}_{+0}^{0,1} \\
\widetilde{\Lambda}_{0-}^{0,1}
\end{array}\right), \\
{ }^{+} \underline{\widetilde{\Lambda}}_{-1}=\underline{\underline{0}}, & +\underline{\widetilde{\Lambda}}^{-1}=\underline{0}, \\
{ }^{Q} \underline{\widetilde{\Lambda}}_{1}=\widetilde{\Lambda}_{-, 1}^{-+}, & { }^{Q} \underline{\widetilde{\Lambda}}^{1}=\widetilde{\Lambda}_{+-1}^{-, 1}, \\
{ }^{Q} \underline{\widetilde{\Lambda}}_{-1}=0, & \underline{\widetilde{\Lambda}}^{-1}=0,
\end{array}
$$

for the ferromagnetic one. The resulting equation for ${ }^{n} \underline{\underline{\Lambda}}_{\alpha}$ reads as:

$$
{ }^{n} \underline{\underline{\Lambda}}_{\alpha}(k)={ }^{n} \underline{\Lambda}_{(0) \alpha}+\int D q{ }^{n} \underline{\underline{\rho}}_{(0)}(q, k+q){ }^{n} \underline{\underline{\Theta}}(q, k+q,-q){ }^{n} \underline{\Lambda}_{(0) \alpha}
$$

for $\alpha= \pm 1$. Where the $\underline{\Lambda}_{(0)}$ vectors are defined similarly as the $\underline{\widetilde{\Lambda}}$ vectors in Eqs. (40) and (41). We have used the consequence of Eq. (31a), namely that $\Theta$ does not depend on its third argument. The solution of Eq. (42) reads as:

$$
{ }^{n} \underline{\underline{\Lambda}}_{\alpha}(k)={ }^{n} \underline{\underline{\varepsilon}}^{\star-1}(k){ }^{n} \underline{\Lambda}_{(0) \alpha}
$$

Similar considerations can be carried on to ${ }^{n} \underline{\widetilde{\Lambda}}^{\alpha}(k)$ with the result:

$$
{ }^{n} \underline{\widetilde{\Lambda}}^{\alpha}(k)={ }^{n} \underline{\underline{\varepsilon}}^{\star-1}(k){ }^{n} \underline{\Lambda}_{(0)}^{\alpha} .
$$

\section{Self-energies in the Hartree-Fock approximation}

Until now we have considered the Hartree-Fock propagators, which are used in the equation of state. The thermal Green's function of the system, defined by Eq. (7) and expressed with the help of the self-energies by the generalized Dyson-Beliaev equation (9), are more complicated. To be consistent both with the partial summation made in the equation of state and the consequences of symmetry breaking, one has to construct the self-energies from the tadpole diagrams in Fig. 5. b) by changing a circle (representing a condensate atom) to an appropriate particle line. The self-energies obtained are diagonal in the upper (Roman) indices; moreover this index is 0 in the polar and + in the ferromagnetic case, i.e. agrees with that of the condensate. The self-energies $\Sigma_{11}^{00}$ and $\Sigma_{11}^{++}$satisfy by construction the relationship:

$$
\frac{1}{\sqrt{N_{0}}} \Sigma_{0,1}^{c}=\Sigma_{11}^{c c}(0,0)-\Sigma_{-1,1}^{c c}(0,0)
$$

where $c=0$ for the polar and $c=+$ for the ferromagnetic case. The Eq. (45) amounts to a generalization of the Hugenholtz-Pines theorem [29] to the spin-1 systems, since

$$
\Sigma_{0,1}^{c}=0, \quad \text { so } \quad \Sigma_{11}^{\prime c c}(0,0)-\Sigma_{-1,1}^{c c}(0,0)=\mu,
$$

where $\Sigma^{\prime}$ is the self-energy without the subtraction $\mu$. This ensures that the corresponding excitations are gapless as required by the general Goldstone theorem due to the breaking of the gauge symmetry.

As for the other self-energies consistency requires that they should be built by using the same diagrammatic blocks and to the same order in them. The resulting Feynman diagrams are depicted in Fig. 7. a) and b). The more detailed diagrammatic equation is shown in appendix A. (Note that in the ferromagnetic phase $\Sigma_{-1,1}^{r s}(k)=0$ except when $r=s=+$ due to the structure of the interaction). One expects that $\Sigma_{11}^{00}(0,0)=0$ in the ferromagnetic phase and $\Sigma_{11}^{--}(0,0)-\Sigma_{-1,1}^{+-}(0,0)=0$ in the polar phase. These relationships lead to gapless spin wave spectra as required by the Goldstone theorem, since the rotational symmetry in the spin space is also broken, besides the gauge symmetry. The spectra to be calculated in the next section will exhibit this property. 


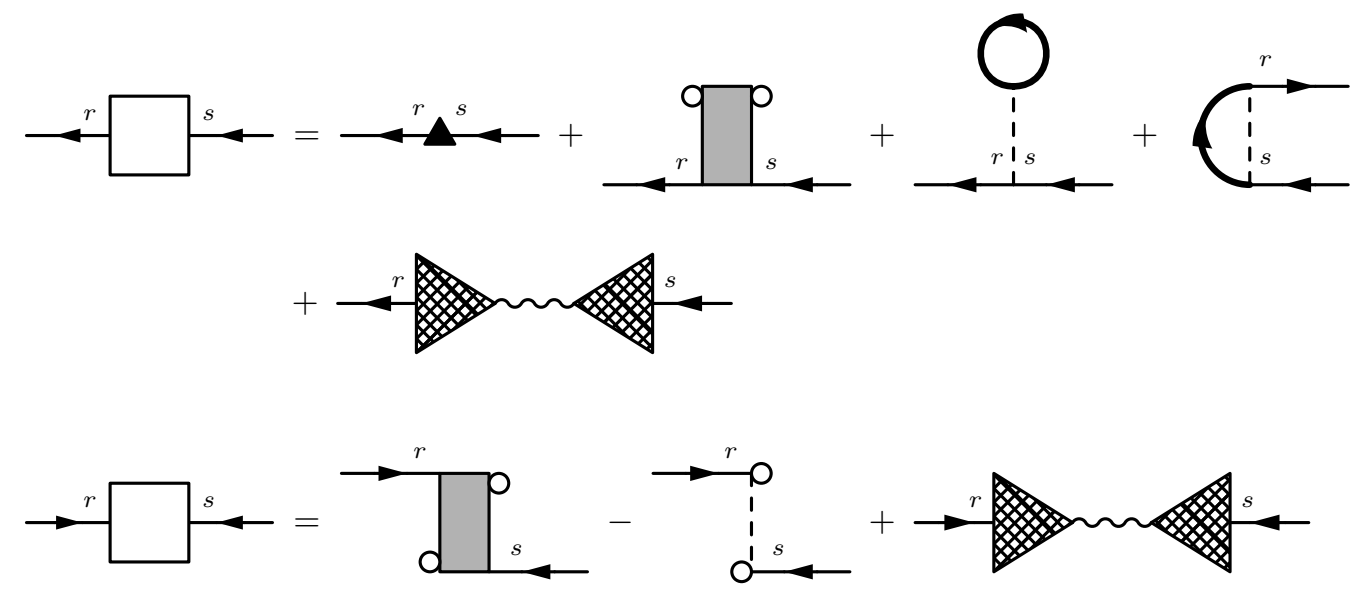

FIG. 7: The Feynman diagrams contributing to the self-energies

According to Fig. 7. a) the self-energy contributions can be brought into two categories. Those graphs are in the first category, which can not be split into two (connected to external lines) by cutting a single interaction line. The first four diagrams fall under this category. We call these self-energy contributions proper. The second category contains those graphs, which do not exhibit this property. The last diagram in Fig. 7. a) falls under this category. We call these latter self-energy contributions improper. Therefore the equation of the normal self-energies read as:

$$
\begin{aligned}
\Sigma_{11}^{r s}(k) & =\widetilde{\Sigma}_{11}^{r s}(k)+M_{11}^{r s}(k), \quad \text { with } \\
\widetilde{\Sigma}_{11}^{r s}(k) & =\hbar^{-1}\left[-\mu+N_{0} \Theta_{r s}^{s^{\prime} r^{\prime}}(0, k, 0)+H^{r^{\prime} s^{\prime}}\left(V_{r s}^{r^{\prime} s^{\prime}}+V_{s^{\prime} s}^{r^{\prime} r}\right)\right], \\
M_{11}^{r s}(k) & =\hbar^{-1} \widetilde{\Lambda}_{c d}^{r, 1}(k) W_{e f}^{d c}(k) \widetilde{\Lambda}_{s, 1}^{f e}(k) .
\end{aligned}
$$

where tilde refers to proper diagrams as in Subsection II C 4. The resulting diagrams for $\Sigma_{-1,1}^{r s}$ are seen in Fig. 7. b) and read as:

$$
\begin{aligned}
& \Sigma_{-1,1}^{r s}(k)=\widetilde{\Sigma}_{-1,1}^{r s}(k)+M_{-1,1}^{r s}(k), \quad \text { with } \\
& \widetilde{\Sigma}_{-1,1}^{r s}(k)=\hbar^{-1} N_{0}\left[\Theta_{r^{\prime} s}^{s^{\prime} r}(-k, 0, k)-V_{r^{\prime} s}^{s^{\prime} r}\right], \\
& M_{-1,1}^{r s}(k)=\hbar^{-1} \widetilde{\Lambda}_{c d}^{r,-1}(k) W_{e f}^{d c}(k) \widetilde{\Lambda}_{s, 1}^{f e}(k) .
\end{aligned}
$$

Finally, for future use we remark that the Green's functions can be categorized according to the amount of spin they transfer relative to the spin of a condensate atom. For this aim we need the following considerations. Equation (9) splits into independent parts according to the amount of spin transfered [19]. For the polar case, let us define the following matrices:

$$
\begin{aligned}
{ }^{0} \mathcal{G}_{\gamma \delta} & =\left[\begin{array}{cc}
\mathcal{G}_{1,1}^{00} & \mathcal{G}_{1,-1}^{00} \\
\mathcal{G}_{-1,1}^{00} & \mathcal{G}_{-1,-1}^{00}
\end{array}\right]_{\gamma \delta}, & { }^{0} \Sigma_{\gamma \delta} & =\left[\begin{array}{cc}
\Sigma_{1,1}^{00} & \Sigma_{1,-1}^{00} \\
\Sigma_{-1,1}^{00} & \Sigma_{-1,-1}^{00}
\end{array}\right]_{\gamma \delta}, \\
{ }^{+} \mathcal{G}_{\gamma \delta} & =\left[\begin{array}{cc}
\mathcal{G}_{1,1}^{--} & \mathcal{G}_{1,-1}^{-+} \\
\mathcal{G}_{-1,1}^{+-} & \mathcal{G}_{-1,-1}^{++}
\end{array}\right]_{\gamma \delta}, & { }^{+} \Sigma_{\gamma \delta} & =\left[\begin{array}{cc}
\Sigma_{1,1}^{--} & \Sigma_{1,-1}^{-+} \\
\Sigma_{-1,1}^{+-} & \Sigma_{-1,-1}^{++}
\end{array}\right]_{\gamma \delta}
\end{aligned}
$$

with the indices in the upper left corner corresponding to the amount of spin transfered. For the ferromagnetic case we define

$$
\begin{aligned}
{ }^{0} \mathcal{G}_{\gamma \delta} & =\left[\begin{array}{cc}
\mathcal{G}_{1,1}^{++} & \mathcal{G}_{1,-1}^{++} \\
\mathcal{G}_{-1,1}^{++} & \mathcal{G}_{-1,-1}^{++}
\end{array}\right]_{\gamma \delta}, & { }^{0} \Sigma_{\gamma \delta} & =\left[\begin{array}{cc}
\Sigma_{1,1}^{++} & \Sigma_{1,-1}^{++} \\
\Sigma_{-1,1}^{++} & \Sigma_{-1,-1}^{++}
\end{array}\right]_{\gamma \delta} \\
{ }^{+} \mathcal{G} & =\mathcal{G}_{1,1}^{00}, & { }^{+}, & =\Sigma_{1,1}^{00} \\
{ }^{Q} \mathcal{G} & =\mathcal{G}_{1,1}^{--}, & Q_{\Sigma} & =\Sigma_{1,1}^{--}
\end{aligned}
$$


With the use of the above matrices, Eq. (9) splits to independent matrix equations, all of the form

$$
{ }^{n} \mathcal{G}_{\alpha \gamma}(k)={ }^{n} \mathcal{G}_{(0) \alpha \gamma}(k)+{ }^{n} \mathcal{G}_{(0) \alpha \rho}(k){ }^{n} \Sigma_{\rho \sigma}(k){ }^{n} \mathcal{G}_{\sigma \gamma}(k),
$$

with $n$ being $0,+$ or $Q$. (Note however, that in the ferromagnetic case for $n=+$ and $n=Q$ anomalous Green's functions do not arise, i.e. $\alpha=\gamma=1$.)

\section{E. Collective excitations and generalized density correlation functions}

To describe collective excitations one can introduce the correlation functions as follows [19]:

$$
\begin{aligned}
& D_{n n}(\mathbf{k}, \tau):=-\left\langle T_{\tau}\left[n(\mathbf{k}, \tau) n^{\dagger}(\mathbf{k}, 0)\right]\right\rangle, \\
& D_{z z}(\mathbf{k}, \tau):=-\left\langle T_{\tau}\left[\mathcal{F}_{z}(\mathbf{k}, \tau) \mathcal{F}_{z}^{\dagger}(\mathbf{k}, 0)\right]\right\rangle, \\
& D_{++}(\mathbf{k}, \tau):=-\left\langle T_{\tau}\left[\mathcal{F}_{+}(\mathbf{k}, \tau) \mathcal{F}_{+}^{\dagger}(\mathbf{k}, 0)\right]\right\rangle, \\
& D_{++}^{Q}(\mathbf{k}, \tau):=-\left\langle T_{\tau}\left[\mathcal{F}_{+}^{Q}(\mathbf{k}, \tau) \mathcal{F}_{+}^{Q \dagger}(\mathbf{k}, 0)\right]\right\rangle,
\end{aligned}
$$

for $k \neq 0$, where the density operator:

$$
n(\mathbf{k})=\sum_{\mathbf{q}} a_{r}^{\dagger}(\mathbf{k}+\mathbf{q}) a_{r}(\mathbf{q})
$$

the spin density operator:

$$
\mathcal{F}_{z}(\mathbf{k})=\sum_{\mathbf{q}} a_{r}^{\dagger}(\mathbf{k}+\mathbf{q})\left(F_{z}\right)_{r s} a_{s}(\mathbf{q})
$$

a non self-adjoint operator generating spin waves:

$$
\mathcal{F}_{+}(\mathbf{k})=\sum_{\mathbf{q}} a_{r}^{\dagger}(\mathbf{k}+\mathbf{q})\left(F_{+}\right)_{r s} a_{s}(\mathbf{q})
$$

and another one for quadrupolar spin waves:

$$
\mathcal{F}_{+}^{Q}(\mathbf{k})=\sum_{\mathbf{q}} a_{r}^{\dagger}(\mathbf{k}+\mathbf{q})\left(F_{+}^{2}\right)_{r s} a_{s}(\mathbf{q})
$$

with $F_{+}=F_{x}+i F_{y}$. After Fourier transformations these correlation functions can be expressed in the following way [19]:

$$
\begin{aligned}
& D_{n n}(k)=\left(\begin{array}{c}
1 \\
1 \\
1
\end{array}\right) \quad{ }^{0} \underline{\underline{D}}(k)\left(\begin{array}{l}
1 \\
1 \\
1
\end{array}\right), \\
& D_{z z}(k)=\left(\begin{array}{c}
1 \\
0 \\
-1
\end{array}\right) \quad \stackrel{\underline{D}}{\underline{0}}(k)\left(\begin{array}{c}
1 \\
0 \\
-1
\end{array}\right), \\
& D_{++}(k)=2\left(\begin{array}{c}
1 \\
1
\end{array}\right)+\underline{\underline{D}}(k)\left(\begin{array}{c}
1 \\
1
\end{array}\right), \\
& D_{++}^{Q}(k)=4{ }^{Q} \underline{\underline{D}}(k) .
\end{aligned}
$$

The matrices on the right hand side satisfy the equations

$$
{ }^{n} \underline{\underline{D}}(k)=\hbar^{n} \underline{\underline{\Pi}}(k)+{ }^{n} \underline{\underline{\Pi}}(k){ }^{n} \underline{\underline{C}}{ }^{n} \underline{\underline{D}}(k),
$$

where ${ }^{n} \underline{\underline{C}}$ has been defined by Eqs. (35) The polarization function can be written as

$$
{ }^{n} \underline{\underline{\Pi}}(k)={ }^{n} \underline{\underline{\Pi}}^{(r)}(k)+\hbar^{-1}{ }^{n} \widetilde{\mathcal{G}}_{\alpha \gamma}(k){ }^{n} \underline{\widetilde{\Lambda}}_{\alpha}(k) \circ{ }^{n} \underline{\widetilde{\Lambda}}^{\gamma}(k) .
$$


Here the first term on the right hand side represents the one-particle line irreducible contributions, which in the Hartree-Fock approximation are given by Eq. (33). Furthermore $\widetilde{\Lambda}$ is defined in the Subsection II C 4 and $\widetilde{\mathcal{G}}$ stands for the proper Green's function obtained as the solution of Eq. (51) with replacing $\Sigma$ by its proper part (given in the Hartree-Fock approximation in Eqs. (47b) and (48b)).

By introducing the so called dielectric function

$$
{ }^{n} \underline{\underline{\varepsilon}}(k)={ }^{n} \underline{\underline{1}}-{ }^{n} \underline{\underline{\Pi}}(k){ }^{n} \underline{\underline{C}},
$$

the frequencies and the damping of collective excitations (after analytic continuation to the second Riemann sheet) can be calculated from the equation

$$
\operatorname{det}{ }^{n} \underline{\underline{\varepsilon}}(\mathbf{k}, \omega)=0,
$$

for $n=\{0,+\}$. For $n=Q$ they are given by the poles of ${ }^{Q} \underline{\underline{\Pi}}$, since ${ }^{Q} \underline{\underline{C}}=0$.

It is important that the one-particle and collective spectra almost coincide as can be proven by rearrangements of the contributions [19]. The spin density relaxation mode in the polar phase, described by the correlation function (54b), is an exception, which does not have a counterpart among the one-particle excitations. One can easily convince oneself that the general treatment in [19] directly applies to the Hartree-Fock model worked out in the present paper.

\section{APPLICATIONS}

In this section we evaluate the self-consistent Hartree-Fock approximation, discussed in the previous section, to parameter values relevant to possible experimental realizations. In most cases we use dimensionless quantities. Let us introduce the following dimensionless small parameters:

$$
\begin{aligned}
\epsilon_{n} & :=\beta_{0} N c_{n}, \\
\epsilon_{s} & :=\beta_{0} N\left|c_{s}\right|, \\
\delta & :=\frac{\left|c_{s}\right|}{c_{n}}=\frac{\epsilon_{s}}{\epsilon_{n}},
\end{aligned}
$$

with $\beta_{0}:=1 /\left(k_{B} T_{0}\right)$ the inverse transition temperature and $N$ the total particle number density. The transition temperature $T_{0}$ is defined as the temperature, where the symmetric phase of the ideal spin-1 Bose gas looses its stability, i.e. where

$$
N=3 \frac{\Gamma\left(\frac{3}{2}\right) F\left(\frac{3}{2}, 0\right)}{4 \pi^{2} \lambda_{0}^{3}}
$$

with $\lambda_{0}:=\lambda\left(T=T_{0}\right)$ the thermal wavelength (15) of the atom at temperature $T_{0}$. The self-energies are made dimensionless with the help of the inverse temperature in the following way:

$$
\sigma_{\alpha \gamma}^{r s}(k):=\beta \hbar \Sigma_{\alpha \gamma}^{r s}(k) .
$$

The condensate density and the thermal densities in their dimensionless form read as:

$$
\begin{aligned}
\gamma_{0} & :=\beta c_{n} N_{0}, \\
C_{r} & :=\beta c_{n} H^{r r}=\frac{\epsilon_{n} \sqrt{t} F\left(\frac{3}{2}, \widehat{\sigma}_{11}^{r r}\right)}{3 \zeta\left(\frac{3}{2}\right)},
\end{aligned}
$$

where $\beta=1 /\left(k_{B} T\right)$ the inverse temperature. Automatic summation is not applied to Eq. (62b), the temperature is also used in a dimensionless form, with $t=T / T_{0}$ and $\zeta(s)$ is the Riemann zeta function. The last equation is obtained with the use of Eq. (14). The dependence on the wavenumber is also evaluated in a dimensionless form. For this aim the thermal wavelength is used again

$$
u:=|\mathbf{k}| \lambda \text {. }
$$

One has to recall at this place that the Hartree-Fock approximation is not applicable in the vicinity of the phase transition. Furthermore, it is not valid at very low temperatures where the contributions of Beliaev processes might become important like in the case of a scalar condensate [30]. Nonetheless there is a considerable temperature range where one can expect that such a Hartree-Fock approach gives quite accurate results. 


\section{A. Ferromagnetic system}

This system is realized by the gas of ${ }^{87} \mathrm{Rb}$ atoms, which has been recently trapped and condensed in an all optical way [31]. As stated earlier, the condensate spinor is chosen to be $\zeta_{r}=(1,0,0)^{T}$. We consider a unit volume system with transition temperature at $T_{0}=200 \mathrm{nK}$ (close to that analytically calculated in Ref. [32] for the experimental situation realized in Ref. [31]), which by Eq. (60) yields a homogeneous total density $N=10^{20} \mathrm{~m}^{-3}$. We take the scattering lengths as $a_{0}=104 a_{\mathrm{B}}$, and $a_{2}=102 a_{\mathrm{B}}$ ( with $a_{\mathrm{B}}$ being the Bohr-radius) according to the calculations made in Ref. [33] ( note that their results have some uncertainty, since the exact number of bound states in the triplet potential of two ${ }^{87} \mathrm{Rb}$ atoms is not known exactly). According to Eqs. (59), the values of the dimensionless small parameters are: $\epsilon_{n}=0.2$ and $\delta=0.0065$.

The length of the condensate is the longest length scale of the system. Taking the length of the condensate as $100 \mu \mathrm{m}$ means a minimal wavenumber $k_{\min }=10^{4} \mathrm{~m}^{-1}$. For experimental applications local excitations should be considered. One can take the length scale of local perturbations to $10 \mu \mathrm{m}$ resulting in a typical wavenumber $k_{\mathrm{typ}}=10^{5} \mathrm{~m}^{-1}$.

With the use of Eqs. (13), (59c), (61), (62) and (17) the dimensionless self-energies of the internal lines read as:

$$
\begin{aligned}
\widehat{\sigma}_{11}^{++} & =(1-\delta) \gamma_{0}, \\
\widehat{\sigma}_{11}^{00} & =-(1-\delta) C_{+}+(1+\delta) C_{0}-2 \delta C_{-}, \\
\widehat{\sigma}_{11}^{--} & =-(1-3 \delta) C_{+}+(1-3 \delta) C_{-}+2 \delta \gamma_{0} .
\end{aligned}
$$

Expressing the condensate density as the total density minus the noncondensate densities leads to the equation:

$$
\gamma_{0}=\frac{\epsilon_{n}}{t}-C_{+}-C_{0}-C_{-} .
$$

Equations (64) and (62b) form a closed set of nonlinear equations for the densities and self-energies , which can be solved numerically.

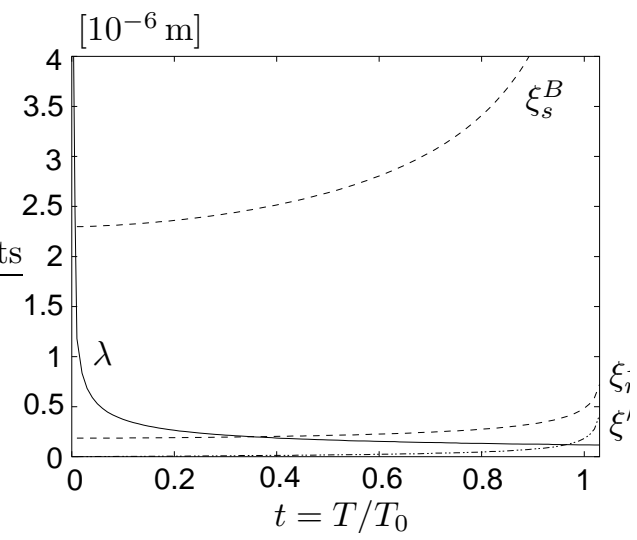

FIG. 8: The characteristic lengths of the ferromagnetic ${ }^{87} \mathrm{Rb}$ system as functions of the dimensionless temperature $t=T / T_{0}$.

\section{Zero spin transfer excitations}

Equations (54a) and (54b) show, that density and spin density collective excitations fall to this category together with the one-particle excitations determined by the zero spin transfer Green's function Eq. (50a). According to Eq. (31a) the matrix ${ }^{n} \underline{\underline{\Theta}}\left(k_{1}, k_{2}, q\right)$ does not depend on $q$, moreover it depends only on $k_{2}-k_{1}$. Using this property and Eqs. (47b), (48b), $\overline{(62}$ ) and (17), the dimensionless proper self-energy of spin-transfer 0 can be brought to the form:

$$
{ }^{0} \widetilde{\sigma}_{\alpha \gamma}(k)=\beta N_{0}\left[\Theta_{++}^{++}(0, k, 0)-\left(c_{n}+c_{s}\right)\right],
$$

for all $\alpha, \gamma$. The zeroth order contributions of the anomalous vertex vectors (41a) read as:

$$
{ }^{0} \underline{\Lambda}_{(0), \alpha}=\sqrt{N_{0}}\left(\begin{array}{l}
1 \\
0 \\
0
\end{array}\right) .
$$


With the help of Eqs. (31a), (31b), (33), (43) and (44) the regular polarization, the anomalous vertex and the proper self-energy can be given explicitly. With the use of these and Eqs. (56) and (57) the dielectric function can be expressed explicitly. The calculation is straightforward but rather lengthy. According to Eq. (58) one way to determine the spectrum of density and spin-density excitations is provided by the equation:

$$
\operatorname{det}{ }^{0} \underline{\underline{\varepsilon}}(\mathbf{k}, \omega)=0 .
$$

Equation (67) can be directly solved numerically. Analytically solvable approximations can also be obtained by keeping the regular terms and the contribution of the nearest pole to the real axis from the Mittag-Leffler representation of the spectral function of the bubble graphs occurring [19]. This approximation is valid for a linear excitational spectrum when the mean-field characteristic lengths are the largest length scales in the system, i.e. when $\xi_{n, s}^{B} \gg \lambda, \xi^{\prime}$. Here $\xi_{n, s}^{B}=\hbar / \sqrt{4 M N_{0}\left|c_{n, s}\right|}$ and $\xi^{\prime}=M /\left(4 \pi \hbar^{2} N_{0} \beta\right)$. The characteristic lengths of the system are depicted in Fig. 8. It is visible that the analytical approximation can be used for $t>0.35$. Both the real and imaginary part of the solution of Eq. (67) is found to depend linearly on momentum for the relevant range. the solution can be written in the form:

$$
\omega=c|\mathbf{k}|-i \Gamma|\mathbf{k}|,
$$

where $c$ is the speed of the excitation and $\Gamma$ is also a positive constant yielding the damping. The numerical and analytical solutions are plotted in Fig. 9. These kind of excitations (density and spin density excitations) show similar features than the density excitations of the scalar gas [17]. They have linear dispersion curve for small wave number with an also linear and small imaginary part.

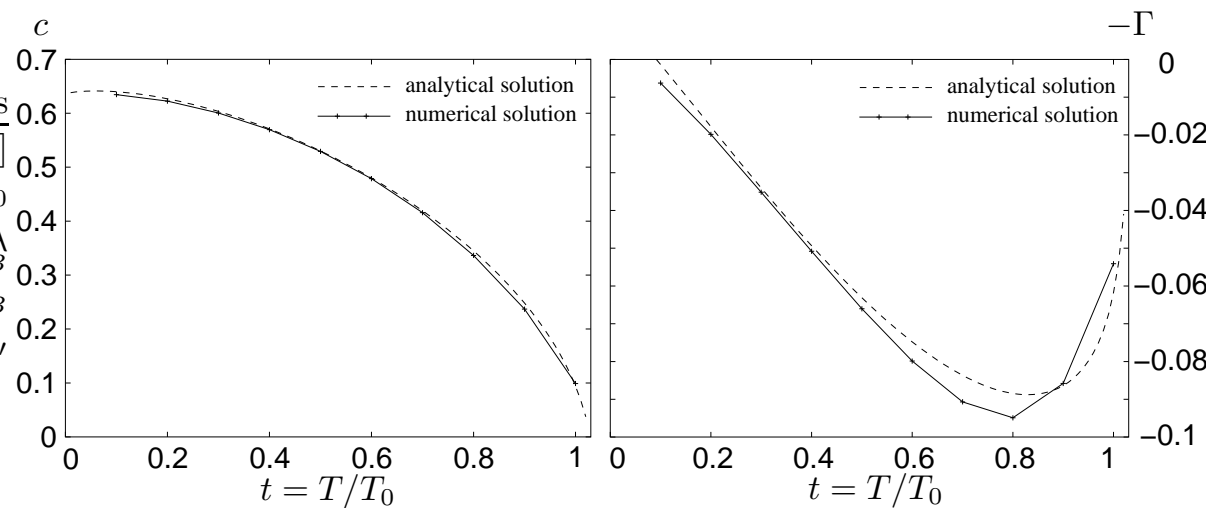

FIG. 9: The sound velocity $c$, and the parameter $-\Gamma$ of the ${ }^{87} \mathrm{Rb}$ ferromagnetic system in units of $k_{B} T_{0} \lambda_{0} / \hbar$, and as the function of $t=T / T_{0}$.

\section{Plus two spin transfer excitations}

As indicated by Eq. (54d) quadrupolar spin waves fall into this category together with the one particle excitations given by the +2 spin transfer Green's function Eq. (50c). Spin conservation forbids here the existence of anomalous Green's functions. Furthermore since ${ }^{Q} C=0$ both the Green's function ${ }^{Q} \mathcal{G}$ and the correlation function ${ }^{Q} D$ are proper. Using Eqs. (47b), (62) and (17), the dimensionless proper self-energy of spin-transfer 2 can be brought to the form:

$$
{ }^{Q} \widetilde{\sigma}_{11}(k)=\beta N_{0}\left[\Theta_{--}^{++}(0, k, 0)-\left(c_{n}+c_{s}\right)\right]+(1-3 \delta)\left(C_{-}-C_{+}\right) .
$$

With the help of Eqs. (28), (31a) and (31b) the $\Theta$ matrix for $n=Q$ can be cast to the form:

$$
\Theta_{--}^{++}(0, k, 0)=\frac{c_{n}(1+\delta)}{1-c_{n}(1+\delta) \Pi_{(0)+-}^{-+}(k)} .
$$

According to Eqs. (51), (61), (69) and (70) the Green's function ${ }^{Q} \mathcal{G}$ is expressed (after the usual analytical continuation in the frequency) as

$$
{ }^{Q} \mathcal{G}(\mathbf{k}, \omega)={ }^{Q} \widetilde{\mathcal{G}}(\mathbf{k}, \omega)=\frac{\beta \hbar}{u^{2}(\Omega-1)-Q \widetilde{\sigma}_{11}(\mathbf{k}, \omega)},
$$


with $u$ defined by Eq. (63), and $\Omega:=\hbar \omega / e_{\mathbf{k}}$. The poles of the Green's function (71) with the use of Eqs. (69), (70) and (62) are determined by the following equation:

$$
u^{2}(\Omega-1)+(1-3 \delta)\left(C_{+}-C_{-}\right)-2 \delta \gamma_{0}-(1+\delta) c_{n} \Pi_{(0)+-}^{-+}(u, \Omega)\left[u^{2}(\Omega-1)+(1-3 \delta)\left(C_{+}-C_{-}\right)+\gamma_{0}(1-\delta)\right]=0 .
$$

Equation (72) can be solved in a numerical way for $\Omega$ as a function of $u$. Analytical approximate solution is also possible with the help of Eqs. (B3) and (B4). If one is also interested in the damping rates, the small imaginary part (B3c) of the bubble graph is also to be considered. The following form is motivated in Appendix B:

$$
c_{n} \mathfrak{I m} \Pi_{(0)+-}^{-+}(u, \Omega)=e^{-\left(\frac{\Delta \hat{\sigma}}{2 u}\right)^{2}}(\ldots), \text { for } \mathfrak{I m} \Omega=0,
$$

with $\Delta \widehat{\sigma}=\widehat{\sigma}_{11}^{--}-\widehat{\sigma}_{11}^{++}$. (We have supposed that the imaginary part of $\Omega$ is very small, therefore $\mathfrak{I m} \Pi=\Pi^{\prime \prime}$.) The quantity in the brackets is not important for our considerations here.

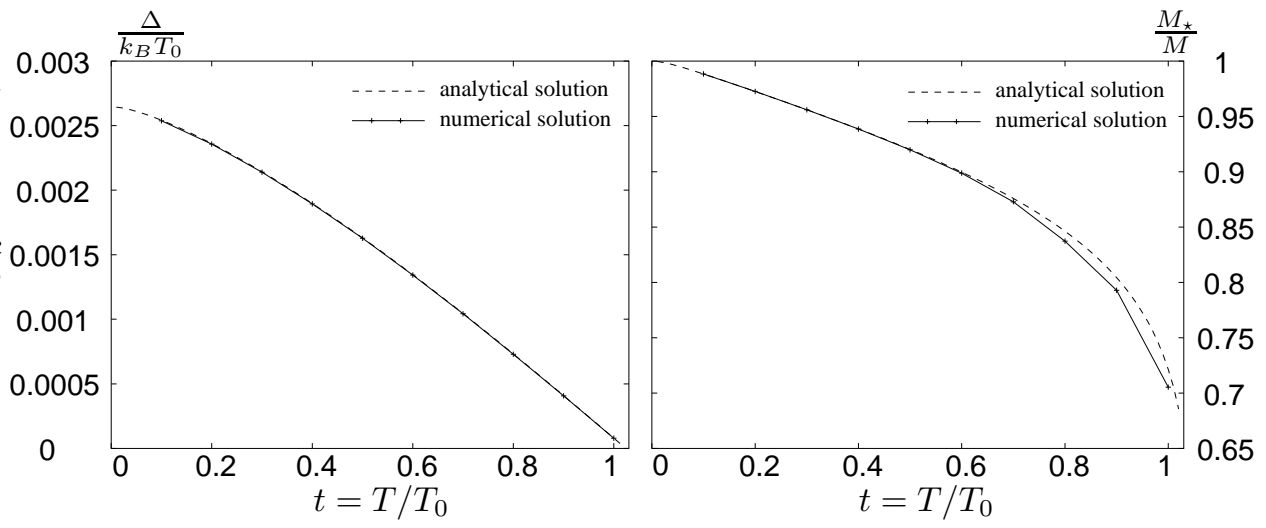

FIG. 10: The gap $\Delta$ and effective mass of the quadrupolar spin wave excitations as functions of the temperature $t=T / T_{0}$. The gap is measured in units of $k_{B} T_{0}$, while $M_{\star}$ is measured in the units of the bare mass of the ${ }^{87} \mathrm{Rb}$ atom.

The long wavelength excitations can be written in the form:

$$
\omega=\frac{\Delta}{\hbar}+\frac{\hbar \mathbf{k}^{2}}{2 M_{\star}}+i \gamma(|\mathbf{k}|)
$$

with $\Delta$ being the energy gap of the quadrupolar excitations, $M_{\star}$ their effective mass and $\gamma(|\mathbf{k}|)$ is their wavenumber dependent damping rate. The gap $\Delta$ and the effective mass $M_{\star}$ is plotted in Fig. 10. The imaginary part of the numerical solution at $T=0.7 T_{0}$ temperature is plotted in Fig. 11. According to Eq. (73) and the numerical solution of Eqs. (64), $\gamma(|\mathbf{k}|)$ vanishes exponentially for $|\mathbf{k}| \rightarrow 0$. This exponential suppression dominates below the characteristic value:

$$
u_{c}=\frac{\left|\widehat{\sigma}_{11}^{--}-\widehat{\sigma}_{11}^{++}\right|}{2} \approx 0.073
$$

For illustration we have fitted the function

$$
f(u)=-C e^{\left(\frac{u_{c}}{u}\right)^{2}}
$$

to the numerical values in Fig. 11 , and found $C \approx 0.089$ and $u_{c} \approx 0.068$ in good agreement with (75).

\section{Plus one spin transfer excitations}

As indicated by Eq. (54c) spin waves fall into this category together with the one particle excitations determined by the +1 spin transfer Green's function Eq. (50b). Spin conservation forbids here the existence of anomalous Green's functions. Using Eqs. (47b), (62) and (17), the dimensionless proper self-energy of spin-transfer 1 can be brought to the form:

$$
{ }^{+} \widetilde{\sigma}_{11}(k)=\beta N_{0}\left[\Theta_{00}^{++}(0, k, 0)-\left(c_{n}+c_{s}\right)\right]-(1-\delta) C_{+}+(1+\delta) C_{0}-2 \delta C_{-} .
$$




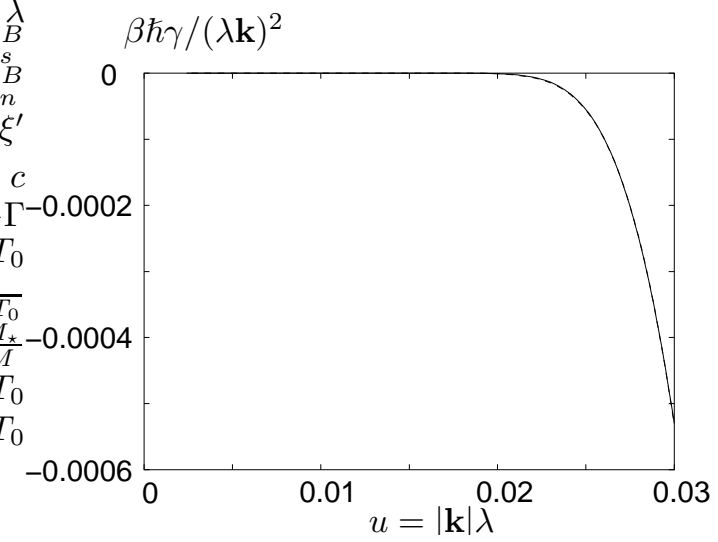

FIG. 11: The damping rate of the quadrupolar spin wave excitations at temperature $T=0.7 T_{0}$. The figure contains the data points of the numerical solution and the curve of the fitting function (76).

The zeroth order contribution for the anomalous vertex vectors Eq. (41b) are

$$
{ }^{+} \underline{\Lambda}_{(0), 1}=\sqrt{N_{0}}\left(\begin{array}{l}
1 \\
0
\end{array}\right), \quad{ }^{+} \underline{\Lambda}_{(0),-1}=\underline{0} .
$$

Again with the help of Eqs. (31a), (31b), (33), (43) and (44) the regular polarization, the anomalous vertex and the proper self-energy can be given explicitly. With the use of these and Eqs. (56) and (57) the dielectric function can be expressed. The spectrum of the spin wave excitations is given as the solution of the equation:

$$
\operatorname{det}+\underline{\underline{\varepsilon}}(\mathbf{k}, \omega)=0
$$

The energy of the excitations is found to be quadratic for small wavenumber (including wavenumber values considered

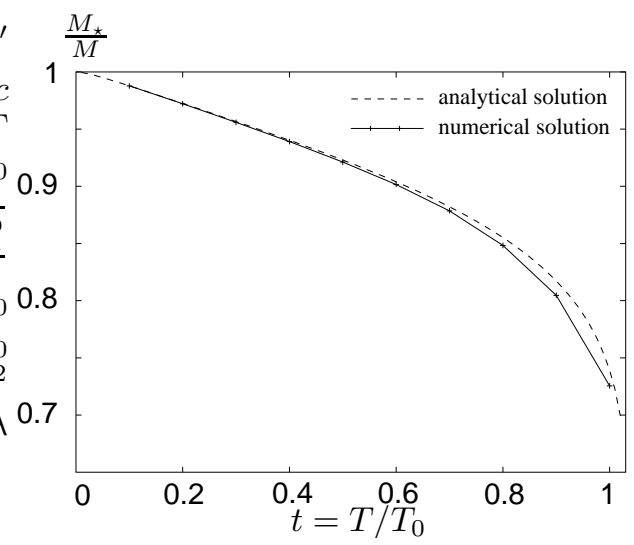

FIG. 12: The effective mass of the spin wave excitations in units of the bare mass of the ${ }^{87} \mathrm{Rb}$ atom as a function of $t=T / T_{0}$.

in this paper). The spectrum of excitations can be written in the form:

$$
\omega=\frac{\hbar \mathbf{k}^{2}}{2 M_{\star}}+i \gamma(|\mathbf{k}|)
$$

with $M_{\star}$ the effective mass and $\gamma(|\mathbf{k}|)$ the damping rate. The temperature dependence of the effective mass is plotted in Fig. 12, while the damping rate is plotted for $T=0.8 T_{0}$ in Fig. 13. The behavior of the damping rate of the spin wave excitations is more complex than that of the quadrupolar spin waves shown in Fig. 11. The reason is that in this case two different bubble graphs emerge. One is $\Pi_{+0}^{0+}$ with self-energy difference $\Delta \widehat{\sigma}_{1}=\widehat{\sigma}_{11}^{00}-\widehat{\sigma}_{11}^{++}$, the other is $\Pi_{0-}^{-0}$ with self-energy difference $\Delta \widehat{\sigma}_{2}=\widehat{\sigma}_{11}^{--}-\widehat{\sigma}_{11}^{00}$. As a consequence there are two characteristic wavenumber values, 


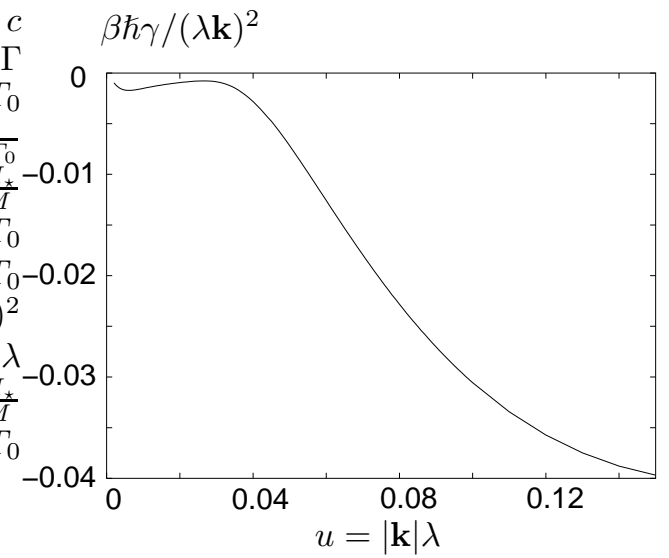

FIG. 13: The damping rate divided by $u^{2}$ of the spin wave excitations in units of $k_{B} T / \hbar$ at $T=0.8 T_{0}$.

where the damping rates suffer a drastic change. At $t=0.8$ these dimensionless wavenumbers are:

$$
\begin{aligned}
& u_{c, 1}=\frac{\left|\widehat{\sigma}_{11}^{--}-\widehat{\sigma}_{11}^{00}\right|}{2} \approx 0.0005, \\
& u_{c, 2}=\frac{\left|\widehat{\sigma}_{11}^{00}-\widehat{\sigma}_{11}^{++}\right|}{2} \approx 0.05 .
\end{aligned}
$$

Since the first wavenumber value is much smaller than the minimal accessible wavenumber $u_{c, 1} \ll \lambda k_{\min }$, there is some damping of the long wavelength spin wave excitations even, when quadrupolar spin waves are not damped.

\section{B. Polar system}

This system is realized by the ultracold gas of ${ }^{23} \mathrm{Na}$ atoms, when the magnetic field is sufficiently small $[3,4]$. We consider a pure condensate and take its spinor as $\zeta_{r}=(0,1,0)^{T}$. The system considered is also of unit volume with periodic boundary conditions and a transition temperature of $T_{0}=2 \mu \mathrm{K}$. The total particle density calculated with Eq. (60) is $N=4.6 \cdot 10^{20} \mathrm{~m}^{-3}$. We take the scattering lengths of the sodium atoms from Ref. [34] to be $a_{0}=50 a_{B}$ and $a_{2}=55 a_{B}$. According to Eqs. (59), the values of the dimensionless small parameters are: $\epsilon_{n}=0.17$ and $\delta=0.031$. To determine the interesting wavenumber range, we also take the condensate size to $100 \mu \mathrm{m}$, from which the minimal wavenumber is $k_{\text {min }}=10^{4} \mathrm{~m}^{-1}$. The length scale of local perturbations is chosen to correspond to a typical wavenumber value $k_{\mathrm{typ}}=10^{5} \mathrm{~m}^{-1}$.

With the use of Eqs. (13), (61), (62) and Eq. (20), and the $(+,-)$ symmetry of the polar case [namely that $\left.\widehat{\sigma}_{11}^{--}=\widehat{\sigma}_{11}^{++}\right]$, the dimensionless self-energies of the internal lines read as:

$$
\begin{aligned}
\widehat{\sigma}_{11}^{00} & =\gamma_{0}, \\
\widehat{\sigma}_{11}^{++} & =(1-\delta)\left(C_{+}-C_{0}\right)+\delta \gamma_{0} .
\end{aligned}
$$

Expressing again the condensate density as the total density minus the noncondensate densities and using the symmetry mentioned leads to the final equation:

$$
\gamma_{0}=\frac{\epsilon_{n}}{t}-2 C_{+}-C_{0}
$$

Equations (82) and (62b) are closed and numerically solvable for the densities and self-energies in the polar phase.

The characteristic lengths of the polar system are plotted in Fig. 14. As can be seen from the figure, the approximation is usable roughly for $t>0.3$ (when the largest characteristic lengths are the mean field correlation lengths).

\section{Zero spin transfer excitations}

As in the case of the ferromagnetic system, these type of excitations are the particle density and spin density excitations defined as the poles of the correlation functions (52a) and (52b) [See Eqs. (54a) and (54b)]. Since the 


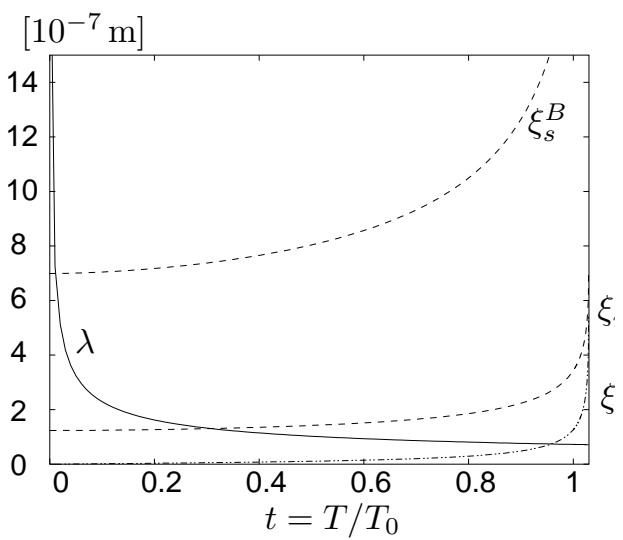

FIG. 14: The characteristic lengths of the polar ${ }^{23} \mathrm{Na}$ system as a function of temperature $t=T / T_{0}$.

$\Theta$ matrix does not depend on the momentum transferred [See the remark after Eqs. (31)], the proper self-energy of spin-transfer 0 for the polar system can be cast to the form (by using Eqs. (47b), (48b), (62) and (20)):

$$
{ }^{0} \widetilde{\sigma}_{\alpha \gamma}(k)=\beta N_{0}\left[\Theta_{00}^{00}(0, k, 0)-c_{n}\right],
$$

for all $\alpha, \gamma$. The zeroth order contributions of the anomalous vertex vectors (40a) read as:

$$
{ }^{0} \underline{\Lambda}_{(0), \alpha}=\sqrt{N_{0}}\left(\begin{array}{l}
0 \\
1 \\
0
\end{array}\right) .
$$

With a lengthy but trivial calculation, using Eqs. (31a), (31b), (33), (43), (44),(56) and (57), one can give the explicite form of the dielectric function ${ }^{0} \underline{\underline{\varepsilon}}\left(\mathbf{k}, i \omega_{n}\right)$. Analytically continuing it in the usual way in the $\omega$ variable one determines the elementary excitations by

$$
\operatorname{det}{ }^{0} \underline{\underline{\varepsilon}}(\mathbf{k}, \omega)=0 \text {. }
$$

It can be seen from the detailed calculation, that the left hand side of Eq. (85) for the polar case is the product of two terms. One term determines the particle density excitations and the other term determines the spin density excitations. This behavior is not limited to this approximation. As shown generally in Ref. [19], the particle density and spin density correlation functions $D_{n n}$ and $D_{z z}$, respectively, have different excitation spectra in the polar case, even if they both belong to spin transfer zero.

The spin density excitations are the solutions of the following equation:

$$
1-(1+3 \delta) c_{n} \Pi_{(0)++}^{++}(u, \Omega)=0
$$

with $\Omega=\hbar \omega / e_{\mathbf{k}}$ and $u=|\mathbf{k}| \lambda$. This is an overdamped mode, its frequency is purely imaginary with linear wavenumber dependence:

$$
\omega=-i \Gamma_{s}|\mathbf{k}|
$$

where $\Gamma_{s}$ is a positive parameter with the dimensionality of velocity. The numerical and analytical solution is plotted in Fig. 15. The analytical solution is obtained from the Mittag-Leffler expansion $[16,19]$ of the spectral function of the bubble graph.

The particle density excitations show the same character as their ferromagnetic counterparts (or as the density excitations in a scalar gas). Namely they have a linear energy spectrum and linear damping rate in the small wavenumber limit. The numerical solution and the analytical approximation from the Mittag-Leffler series [16, 19] is plotted in Fig. 16, where $c$ and $\Gamma$ are defined as in Eq. (68). 


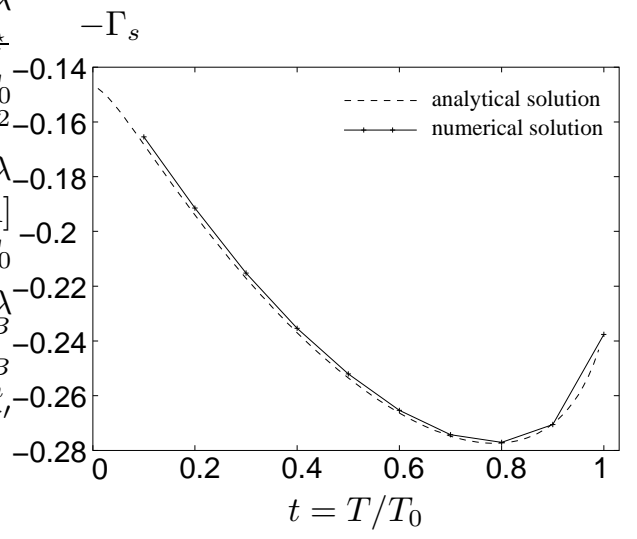

FIG. 15: The relaxation rate parameter $-\Gamma_{s}$ of the spin density wave of the ${ }^{23} \mathrm{Na}$ (polar) system in units of $k_{B} T_{0} \lambda_{0} / \hbar$, and as the function of $t=T / T_{0}$.
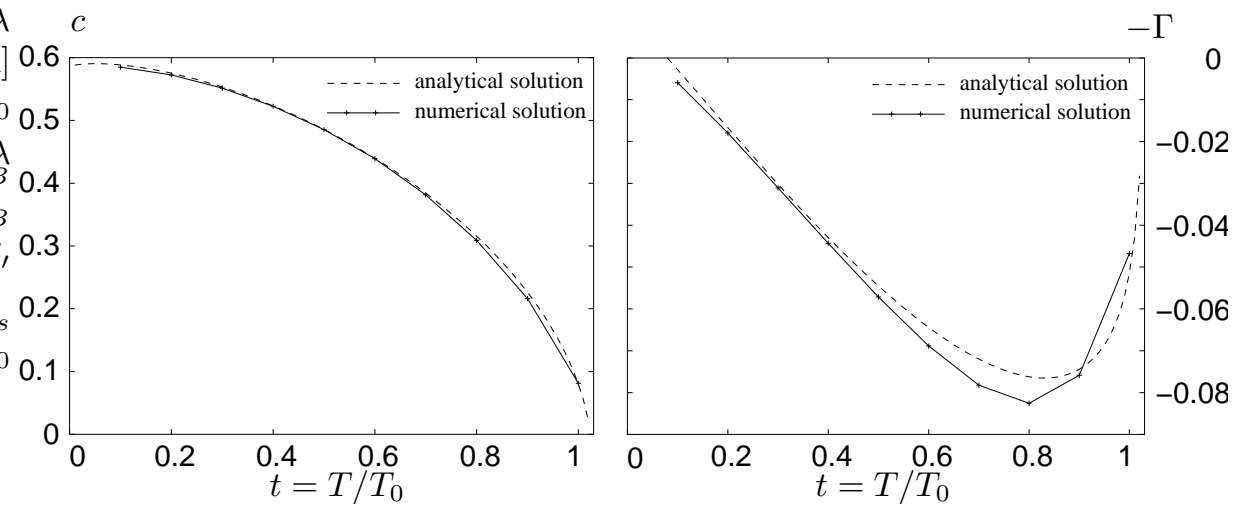

FIG. 16: The sound velocity $c$, and the parameter $-\Gamma$ of the particle density excitations of the ${ }^{23} \mathrm{Na}$ (polar) system in units of $k_{B} T_{0} \lambda_{0} / \hbar$, and as the function of $t=T / T_{0}$.

\section{Plus one spin transfer excitations}

The +1 spin transfer excitations are spin waves, as shown by Eq. (54c). Using Eqs. (47b), (62) and (20), the dimensionless proper self-energy of spin-transfer 1 can be brought to the form:

$$
\begin{aligned}
+\widetilde{\sigma}_{11}(k) & =\beta N_{0}\left[\Theta_{--}^{00}(0, k, 0)-c_{n}\right]+(1-\delta)\left(C_{+}-C_{0}\right), \\
+\widetilde{\sigma}_{1,-1}(k) & =\beta N_{0}\left[\Theta_{-0}^{+0}(0, k,-k)-c_{s}\right], \\
+\widetilde{\sigma}_{-1,1}(k) & =\beta N_{0}\left[\Theta_{0-}^{0+}(-k, 0, k)-c_{s}\right], \\
+\widetilde{\sigma}_{-1,-1}(k) & =\beta N_{0}\left[\Theta_{00}^{++}(-k, 0,0)-c_{n}\right]+(1-\delta)\left(C_{+}-C_{0}\right) .
\end{aligned}
$$

The zeroth order contribution for the anomalous vertex vectors Eq. (40b) are

$$
{ }^{+} \underline{\Lambda}_{(0), 1}=\sqrt{N_{0}}\left(\begin{array}{l}
0 \\
1
\end{array}\right), \quad{ }^{+} \underline{\Lambda}_{(0),-1}=\sqrt{N_{0}}\left(\begin{array}{l}
1 \\
0
\end{array}\right) .
$$

Again with the help of Eqs. (31a), (31b), (33), (43) and (44) the regular polarization, the anomalous vertex and the proper self-energy can be given explicitly. With the use of these and Eqs. (56) and (57) the dielectric function can be expressed. The spin wave excitations of the polar state are given by the equation:

$$
\operatorname{det}{ }^{+} \underline{\underline{\varepsilon}}(\mathbf{k}, \omega)=0 \text {. }
$$

Since the $\Pi_{(0)+0}^{0+}$ and $\Pi_{(0) 0-}^{-0}$ bubbles appear in Eq. (93), the analytical solution is also obtained from the asymptotic series expansion (B4) of the spectral function of the bubble graph. 
In the polar case $\widehat{\sigma}_{11}^{++}=\widehat{\sigma}_{11}^{--}$, therefore the self energy difference of the internal lines for the two bubbles are equal, and as a consequence the damping rate of the spin wave excitations will be exponentially suppressed but with one characteristic wavenumber value: $u_{c}=\left|\widehat{\sigma}_{11}^{00}-\widehat{\sigma}_{11}^{++}\right| / 2$. The spectrum of spin wave excitations in the polar case can be written in the form:

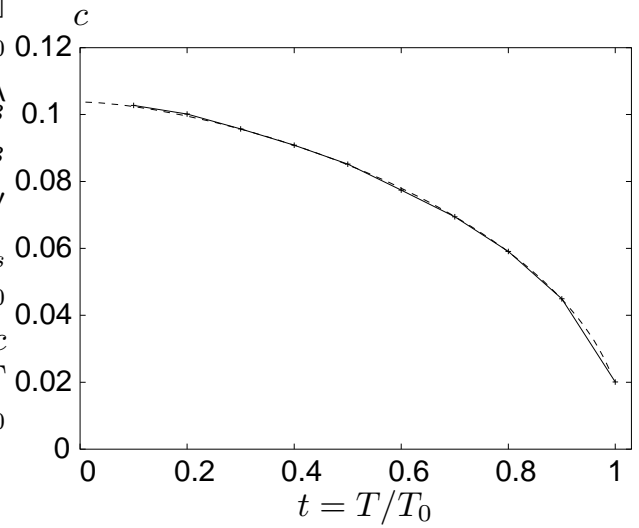

FIG. 17: The velocity of the spin-wave excitations of the ${ }^{23} \mathrm{Na}$ (polar) system in units of $k_{B} T_{0} \lambda_{0} / \hbar$, and as the function of $t=T / T_{0}$.

$$
\omega=c|\mathbf{k}|+i \gamma(|\mathbf{k}|)
$$

The numerical and analytical solutions for the sound velocity of the spin wave excitations are to be found in Fig. 17. The damping rate is plotted for $T=0.8 T_{0}$ in Fig. 18 .

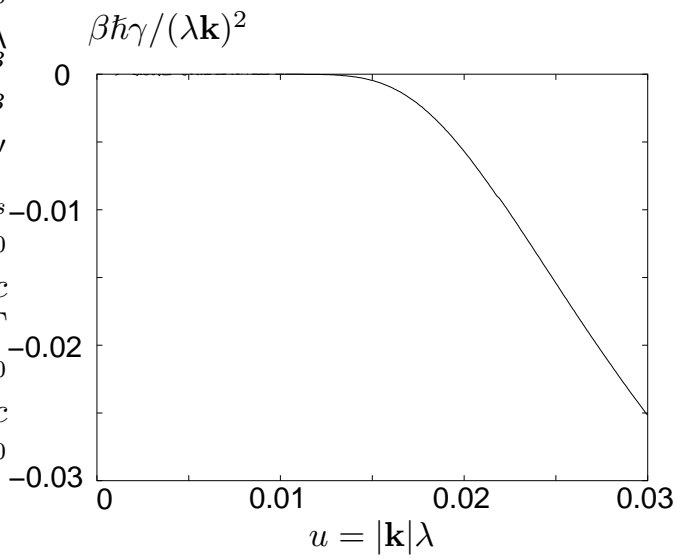

FIG. 18: The damping rate of the spin wave excitations at temperature $T=0.8 T_{0}$ for the ${ }^{23} \mathrm{Na}$ (polar) system in units of $(k \lambda)^{2} /(\hbar \beta)$, and as the function of $u=|\mathbf{k}| \lambda$.

As a summary we compare the frequencies and damping rates of the various excitations of both the ferromagnetic and polar cases for a given momentum value, namely $k=k_{\text {typ }}=10^{5} \mathrm{~m}^{-1}$. In Fig. 19. the excitation frequencies and damping rates of the collective excitations of the ferromagnetic system are plotted. It can be seen, that for this wavenumber value, density excitations (density fluctuations) cost much more energy and are relatively shortlived compared to spin waves and quadrupolar spin waves. These latter excitations correspond to transverse spin fluctuations of the system with constant density. In Fig. 20. the frequencies and damping rates of the excitations of the polar system are shown. Density fluctuations cost more energy than transverse spin fluctuations (in the polar case, we mean transverse as orthogonal to the spin quantization axis) in this case too and are also shorter lived than the latter ones. The shortest living excitations in the polar case however are the non-propagating spin density excitations, which belong to longitudinal spin fluctuations. 

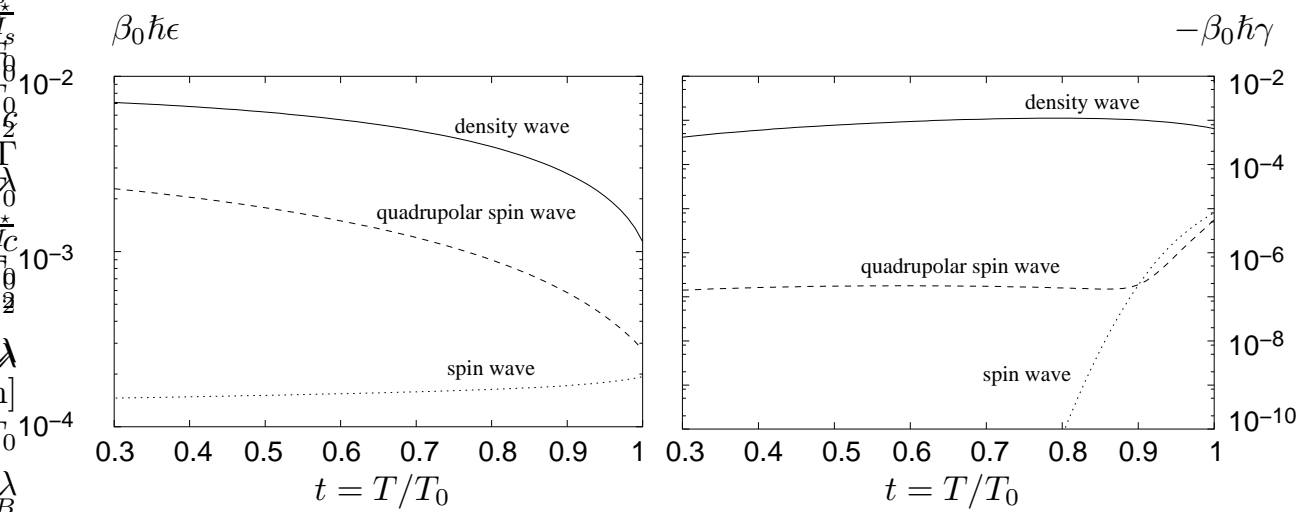

FIG. 19: The frequencies $(\epsilon=\mathfrak{R e} \omega)$ and damping rates $(\gamma=\mathfrak{I m} \omega)$ as functions of dimensionless temperature $t=T / T_{0}$ of the various collective excitations for $k=k_{\mathrm{typ}}=10^{5} \mathrm{~m}^{-1}$ of the ferromagnetic system. Both quantities are measured in units of $k_{B} T_{0} / \hbar$.
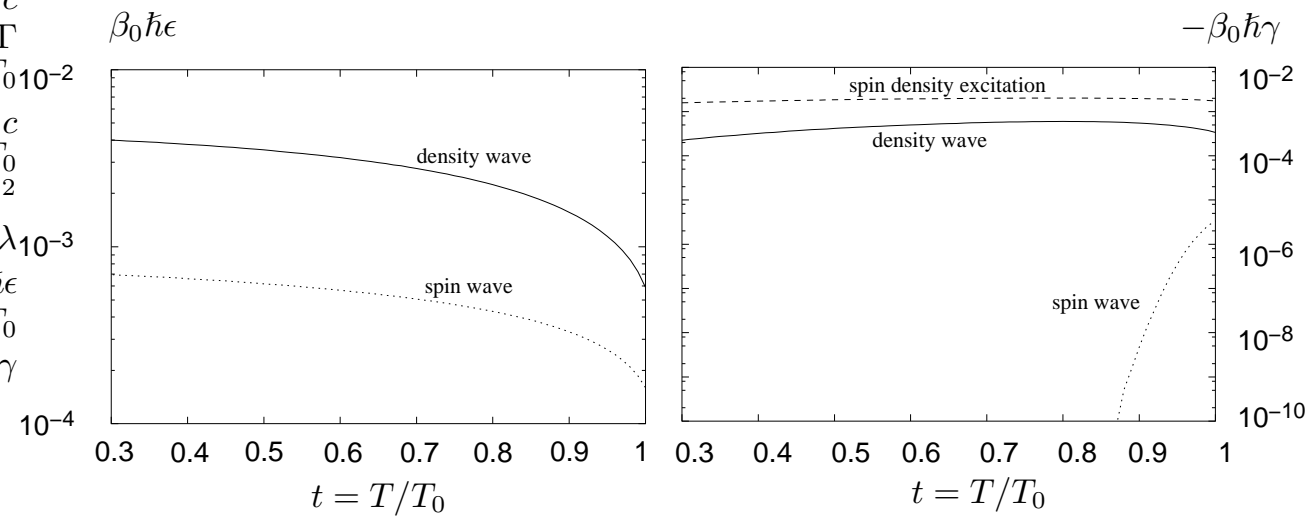

FIG. 20: The frequencies $(\epsilon=\mathfrak{R e} \omega)$ and damping rates $(\gamma=\mathfrak{I m} \omega)$ as functions of dimensionless temperature $t=T / T_{0}$ of the various collective excitations for $k=k_{\mathrm{typ}}=10^{5} \mathrm{~m}^{-1}$ of the polar system. Both quantities are measured in units of $k_{B} T_{0} / \hbar$.

\section{DISCUSSION}

In this paper we have generalized the self-consistent Hartree-Fock theory to the symmetry breaking phase of the spin-1 Bose gas. We have started from the Hartree-Fock equation of state of the ferromagnetic and polar condensed phases. The Green's functions and correlation functions have been given, in accordance with the self-consistent equation of state and with the dielectric formalism, from which the one-particle and collective excitations of the system have been determined. The results have been applied to the cases of ${ }^{23} \mathrm{Na}$ (polar) and ${ }^{87} \mathrm{Rb}$ (ferromagnetic) gases.

Both in the ferromagnetic and polar cases the behavior of particle density excitations, belonging to spin transfer 0 , has been found similar to that of the particle density waves of scalar Bose gases, where these are the solely existing collective modes. In particular both their frequency and damping rate depend linearly on wavenumber, see Eq. (68), Figs. 9. and 16., and Ref. $[12,16,17,19]$ in the intermediate temperature region. These collective excitations agree with one-particle excitations belonging to spin transfer 0 due to hybridization caused by the condensate. In the polar case also for spin transfer 0 another collective mode (independent of particle density waves) exists, which is overdamped (Fig. 15.) and represents spin relaxation. This collective mode is related to the pole of the correlation function (52b). Furthermore, it is absent from the one-particle excitations [19], which is the consequence of the $+\leftrightarrow-$ spin inversion symmetry of the polar phase and is independent of the approximation. Spin waves are described by the correlation functions (52c) and are collective excitations with spin transfer 1 . They hybridize with the one-particle excitations with the same spin transfer in both the ferromagnetic and polar cases. It has been found that with taking into account the exchange processes the properties of these excitations calculated in Ref. [19] essentially change. In the ferromagnetic case, though the excitation energies continue to show a quadratically starting energy spectrum, 
the behavior of the effective mass as a function of temperature (Fig. 12.) differs qualitatively from that of the same excitations calculated in the Hartree approximation, which contains only direct interaction processes. Note, that in the Bogoliubov approximation the effective mass has been the bare one [8, 9]. In the polar case the excitation frequencies depend linearly on wavenumber with a velocity depicted on Fig. 17. This feature has been found already in the Bogoliubov and the Hartree approximations $[8,9,19]$. In these cases, however, the interaction parameter $c_{n}$ does not show up in the velocity of the spin wave. It is no more the case in the present Hartree-Fock approach and the solution drawn on Fig. 17. includes it. The imaginary part of the spectrum, i.e. the damping rate is, however, exponentially suppressed (Fig. 18.), differing qualitatively from the damping rate in the Hartree approximation, which is linearly dependent on wavenumber in the relevant temperature region. The reason of the observed significant difference is that, while in the Hartree approximation all three self-energies belonging to internal lines with different spin projection are zero, in the Hartree-Fock approximation only the self-energies of the $\widehat{\mathcal{G}}_{11}^{++}$and $\widehat{\mathcal{G}}_{11}^{--}$propagators are equal in the polar case. This latter coincidence follows from the $+\leftrightarrow-$ symmetry of the polar state. The lift of the degeneration results in the appearance of the characteristic wavenumber under which the damping rate of the excitations are suppressed. (In the ferromagnetic case all three internal lines have different self-energies, therefore the damping rate of both the spin wave and quadrupolar spin wave are suppressed for small momentum values, see Figs. 11. and 13.) This can be regarded as a threshold effect. One expects that in the intermediate temperature region its occurrence is not restricted to the Hartree-Fock approximation, but it is generally present though can be less pronounced. The reason is that in this temperature region scattering processes at wavenumber greater than the inverse of the Bogoliubov coherence length make the important contributions and the use of the Hartree-Fock propagator for the internal lines is justified. Collective excitations with spin transfer 2 (quadrupolar excitations) are propagating only in the ferromagnetic case. The frequency of these excitations start with a gap (since these are non Goldstone excitations) and is parabolic (see Fig. 10.). Its effective mass is close to that of the ferromagnetic spin waves, the difference is proportional to the smaller coupling constant $c_{s}$. The existence of a gap and the quadratic dispersion has been found already in the Bogoliubov and Hartree approximations [8, 9, 19].

Some remarks concerning hybridization of one-particle and collective excitations are in order here. We have used this terminology in the sense as introduced in case of a scalar condensate. Namely, that due to the presence of the condensate one can see intermediate states corresponding to collective excitations in the perturbation series of the one-particle propagators and similarly, one can identify one-particle intermediate states in the perturbation series of the correlation functions describing collective modes. As a result the one-particle and the collective excitations spectra coincide after the necessary rearrangement of the perturbation series, carried out within the framework of the so called dielectric formalism (see for a review Ref. [13]). In case of the systems with a spinor condensate there are exceptions when such hybridization does not take place [19]. One example is the spin density relaxation in the polar phase described by the correlation function $D_{z z}(52 \mathrm{~b})$, whose perturbation series does not contain one-particlelike intermediate states (see Eqs. (86), (87)). Another example is provided by the quadrupolar excitations in the ferromagnetic phase. Though in the perturbation series $D_{++}^{Q}(52 \mathrm{~d})$ one-particle like intermediate states occur, it is not true vice versa since the Green's function is proper in this case (see III A 2. chapter of the Applications). As a result the spectra of the correlation functions (52) is more complete than those of the Green's functions. A further advantage of using the correlation functions is that the physical nature of the excitation calculated can be seen from the definition of the relevant correlation function. By these reasons we also have put emphasis on them in the Section devoted to the applications.

We should also comment on the region of validity of the self-consistent Hartree-Fock approximation. This approximation can not be used very close to zero temperature. In this regime other processes give significant contributions to the self-energies (Beliaev processes). The Hartree-Fock approximation is expected to provide quantitatively good results when the mean-field coherence lengths are the longest length scales in the system. It can be seen in Figs. 8. and 14 . that this is the case above $T \approx 0.3 T_{0}$. In this model approximation it is found that the transition is a first order one for both the ferromagnetic and polar cases, such as for the scalar gas [17]. On the other hand the transition is proved to be second order for the polar case and weakly first order for the ferromagnetic case in the self-consistent Hartree approximation [19]. Since both approximations represent improved mean-field approaches they are not applicable near the transition point, where a more sophisticated treatment is needed, which can also decide upon the order of transition.

\section{ACKNOWLEDGEMENT}

The present work has been partially supported by the Hungarian Research National Foundation under grant No. OTKA T029552. 


\section{APPENDIX A: THE SELF-ENERGIES IN THE HARTREE-FOCK APPROXIMATION}

In this appendix we show how the self-energies $\Sigma_{11}^{c c}$ and $\Sigma_{-1,1}^{c c}$ (with $c=0$ for the polar and $c=+$ for the ferromagnetic phase), depicted in general in Fig. 7., can be obtained by changing all condensate circles to external lines, with the same spin projection, in the tadpole diagrams Fig. 5. b). The result of the above process is illustrated in Fig. 21. With the help of the diagramatic equation in Fig. 5. a) one can replace the self-energy contribution of the internal line by the simpler form depicted in Fig. 1. Also using the anomalous vertex vectors, plotted in Fig. 6 one arrives at the self-energies as illustrated in Fig. 7.
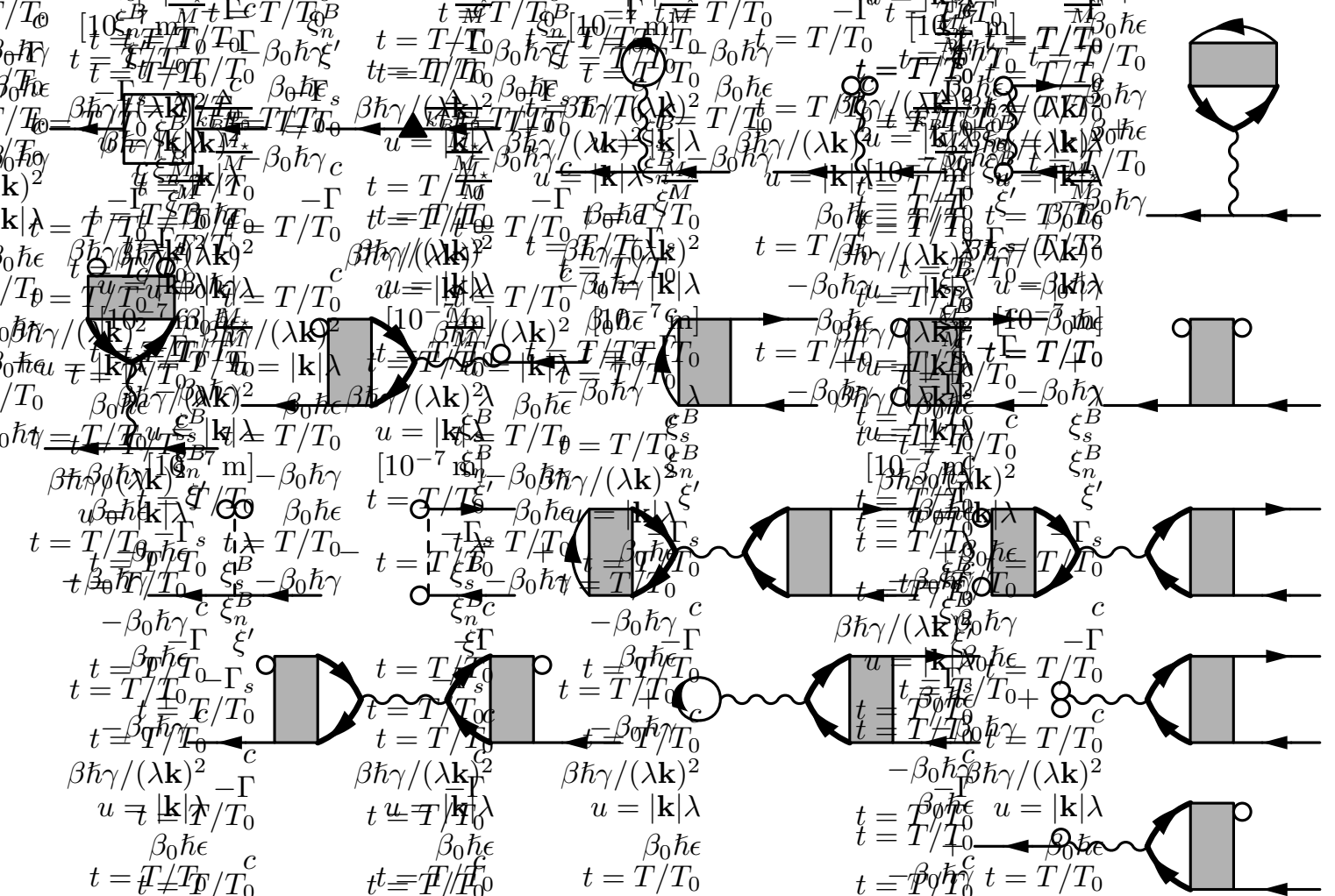

(a)

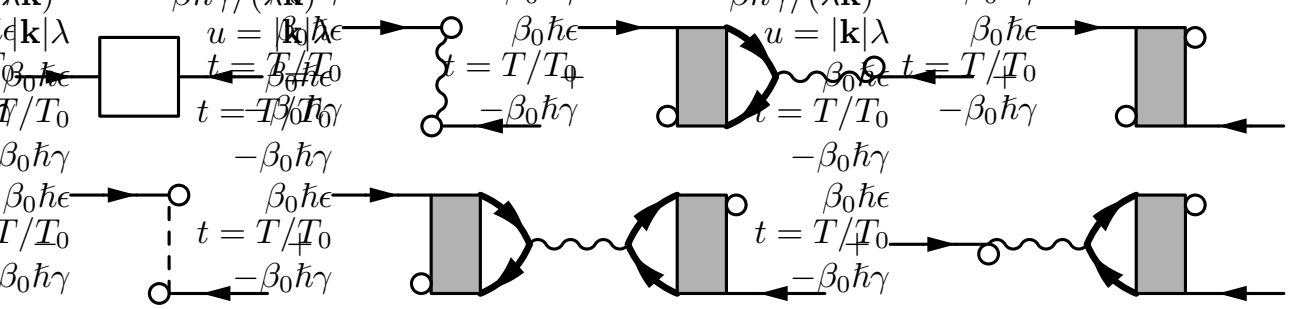

(b)

FIG. 21: The structure of the self-energies $\Sigma_{11}^{c c}$ and $\Sigma_{-1,1}^{c c}$

\section{APPENDIX B: THE ANALYTICAL APPROXIMATION OF THE BUBBLE GRAPHS WITH DIFFERENT SELF-ENERGIES}

In this appendix we outline the approximation of the bubble graphs with different self-energies of the propagators. The contribution of the analytically continued bubble graph, with internal propagators carrying spin $s$ and spin $r$, forward and backward, respectively, can be cast to the form [19]:

$$
c_{n} \Pi_{(0) r s}^{s r}(u, \Omega)=\frac{\epsilon_{n} \sqrt{t}}{12 \Gamma(3 / 2) \zeta(3 / 2) u}\left[\int_{\infty}^{y_{0}} R_{\widehat{\sigma}_{11}^{r r}}(z) d z-\int_{\infty}^{y_{1}} R_{\widehat{\sigma}_{11}^{s s}}(z) d z\right],
$$


with the notations

$$
\begin{aligned}
y_{0} & =\frac{1}{2 u}\left[u^{2}(\Omega-1)-\Delta \widehat{\sigma}\right], \\
y_{1} & =\frac{1}{2 u}\left[u^{2}(\Omega+1)-\Delta \widehat{\sigma}\right], \quad \text { and } \\
\Delta \widehat{\sigma} & =\widehat{\sigma}_{11}^{s s}-\widehat{\sigma}_{11}^{r r} .
\end{aligned}
$$

The function

$$
R_{\widehat{\sigma}}(z)=\int_{-\infty}^{\infty} \frac{2 x}{e^{x^{2}+\widehat{\sigma}}-1} \frac{1}{x-z} d x, \quad(\text { for } \quad \mathfrak{I m} z>0)
$$

(analytical in the upper half-plane) is to be continued to the whole complex plane. We are interested in the $\mathfrak{I m} \Omega<0$ values, since the elementary excitations have finite lifetimes. Therefore $z$ also has a negative imaginary part, therefore the function reads as:

$$
\begin{aligned}
R_{\widehat{\sigma}}(z) & =R_{\widehat{\sigma}}^{\prime}(z)+R_{\widehat{\sigma}}^{\prime \prime}(z), \quad \text { with } \\
R_{\widehat{\sigma}}^{\prime}(z) & =\int_{-\infty}^{\infty} \frac{2 x}{e^{x^{2}+\widehat{\sigma}}-1} \frac{1}{x-z} d x \\
R_{\widehat{\sigma}}^{\prime \prime}(z) & =\frac{4 i \pi z}{e^{z^{2}+\widehat{\sigma}}-1}, \quad(\text { for } \quad \mathfrak{I m} z<0) .
\end{aligned}
$$

In the long wavelength limit, i.e. when $2 u \ll|\Delta \widehat{\sigma}|$, the upper limits of the integrals in Eq. (B1a) diverge, therefore an asymptotic series expansion can be used to represent the integral Eq. (B3b):

$$
R_{\widehat{\sigma}}^{\prime}(z)=-\frac{2}{z^{2}} \sum_{n=0}^{\infty} \frac{\Gamma(n+3 / 2) F(n+3 / 2, \widehat{\sigma})}{z^{2 n}} .
$$

Let us define the quantities $\Pi_{(0) r s}^{\prime s r}$ and $\Pi_{(0) r s}^{\prime \prime}$, with Eq. (B1) by replacing $R$ with $R^{\prime}$, and $R^{\prime \prime}$, respectively. One obtains

$$
c_{n} \Pi_{(0) r s}^{\prime \prime s r}(u, \Omega)=\frac{2 \pi i \epsilon_{n} \sqrt{t}}{12 \Gamma(3 / 2) \zeta(3 / 2) u} \ln \frac{1-e^{-y_{0}^{2}+\widehat{\sigma}_{11}^{r r}}}{1-e^{-y_{1}^{2}+\widehat{\sigma}_{11}^{s s}}} .
$$

In the same limit, i.e. when $2 u \ll|\Delta \widehat{\sigma}|$, one can further approximate $c_{n} \Pi_{(0) r s}^{\prime \prime}$ with a result:

$$
\begin{array}{r}
c_{n} \Pi_{(0) r s}^{\prime \prime s r}(u, \Omega)=\frac{2 \pi i \epsilon_{n} \sqrt{t}}{12 \Gamma(3 / 2) \zeta(3 / 2) u} e^{-\frac{\Delta \widehat{\sigma}^{2}}{4 u^{2}}}\left[\exp \left(-\frac{u^{2}(\Omega-1)^{2}-2 \Delta \widehat{\sigma}(\Omega-1)-4 \widehat{\sigma}_{11}^{s s}}{4}\right)\right. \\
\left.-\exp \left(-\frac{u^{2}(\Omega+1)^{2}-2 \Delta \widehat{\sigma}(\Omega+1)-4 \widehat{\sigma}_{11}^{r r}}{4}\right)\right] .
\end{array}
$$

Taking the real part of $\Omega$ positive, and with the assumption that its imaginary part is small one can see that the quantity in the square brackets is not important for the considerations made in Sec. III.

We have considered the analytical approximation of the retarded part of the bubble graph's contribution with differing self-energies in the forward and backward propagating lines, i.e. with nonzero spin transfer. The approximation for such bubble graphs with the same self-energies in their internal lines, i.e. those occurring in zero spin transfer processes, are obtained by a Mittag-Leffler expansion, discussed in detail e.g. in Ref. [16].

[1] M. H. Anderson, J. R. Ensher, M. R. Matthews, C. E. Wieman, and E. A. Cornell, Science 269, 198 (1995).

[2] K. B. Davis, M.-O. Mewes, M. R. Andrews, N. J. van Druten, D. S. Durfee, D. M. Kurn, and W. Ketterle, Phys. Rev. Lett. 75, 3969 (1995).

[3] D. M. Stamper-Kurn, M. R. Andrews, A. P. Chikkatur, S. Inouye, H.-J. Miesner, J. Stenger, and W. Ketterle, Phys. Rev. Lett. 80, 2027 (1998). 
[4] J. Stenger, D. M. Stamper-Kurn, M. R. Andrews, A. P. Chikkatur, S. Inouye, H.-J. Miesner, and W. Ketterle, J. Low Temp. Phys. 113, 167 (1998).

[5] J. Stenger, S. Inouye, D. M. Stamper-Kurn, H.-J. Miesner, A. P. Chikkatur, and W. Ketterle, Nature 396, 345 (1999).

[6] H.-J. Miesner, D. M. Stamper-Kurn, J. Stenger, S. Inouye, A. P. Chikkatur, and W. Ketterle, Phys. Rev. Lett. 82, 2228 (1999).

[7] D. Stamper-Kurn and W. Ketterle, in Les Houches, Session LXXII, Coherent atomic matter waves, edited by R. Kaiser, C. Westbrook, and F. David (EDP Sciences; Springer-Verlag, Les Ulis; Berlin, 2001), p. 137.

[8] T.-L. Ho, Phys. Rev. Lett. 81, 742 (1998).

[9] T. Ohmi and K. Machida, J. Phys. Soc. Jpn. 67, 1822 (1998).

[10] N. Bogoliubov, J. Phys. 11, 23 (1947).

[11] S. T. Beliaev, Sov. Phys. JETP 34, 299 (1958).

[12] H. Shi and A. Griffin, Phys. Rep. 304, 1 (1998).

[13] A. Griffin, Excitations in a Bose-condensed liquid (Cambridge University Press, Cambridge, 1993).

[14] A. Griffin, Phys. Rev. B. 53, 9341 (1996).

[15] V. N. Popov, Functional Integrals in Quantum Field Theory and Statistical Physics (Reidel, Dordrecht, 1983).

[16] P. Szépfalusy and I. Kondor, Ann. Phys. (N.Y.) 82, 1 (1974).

[17] M. Fliesser, J. Reidl, P. Szépfalusy, and R. Graham, Phys. Rev. A 64, 013609 (2001).

[18] P. Szépfalusy and G. Szirmai, Phys. Rev. A 61, 051604(R) (2000).

[19] P. Szépfalusy and G. Szirmai, Phys. Rev. A 65, 043602 (2002).

[20] A. Minguzzi and M. Tosi, J. Phys. Condens. Matter 9, 10211 (1997).

[21] E. J. Mueller and G. Baym, Phys. Rev. A 62, 053605 (2000).

[22] J. Reidl, A. Csordás, R. Graham, and P. Szépfalusy, Phys. Rev. A 61, 043606 (2000).

[23] M. R. Andrews, D. M. Kurn, H.-J. Miesner, D. S. Durfee, C. G. Townsend, S. Inouye, and W. Ketterle, Phys. Rev. Lett. 79, 553 (1997).

[24] G. Bene and P. Szépfalusy, Phys. Rev. A 58, R3391 (1998).

[25] J. Reidl, G. Bene, R. Graham, and P. Szépfalusy, Phys. Rev. A 63, 043605 (2001).

[26] M. Bijlsma and H. T. C. Stoof, Phys. Rev. A 55, 498 (1997).

[27] A. Fetter and J. Walecka, Quantum Theory of Many-Particle Systems (McGrow-Hill, New York, 1971).

[28] J. E. Robinson, Phys. Rev. 83, 678 (1951).

[29] N. M. Hugenholtz and D. Pines, Phys. Rev. 116, 489 (1959).

[30] P. Fedichev and G. Shlyapnikov, Phys. Rev. A 58, 3146 (1998).

[31] M. D. Barrett, J. A. Sauer, and M. S. Chapman, Phys. Rev. Lett. 87, 010404 (2001).

[32] W.-J. Huang, S.-C. Gou, and Y.-C. Tsai, Phys. Rev. A 65, 063610 (2002).

[33] N. N. Klausen, J. L. Bohn, and C. H. Greene, Phys. Rev. A 64, 053602 (2001).

[34] A. Crubellier, O. Dulieu, F. Masnou-Seeuws, M. Elbs, H. Knckel, and E. Tiemann, Eur. Phys. J. D 6, 211 (1999). 